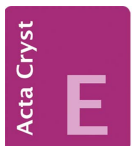

CRYSTALLOGRAPHIC COMMUNICATIONS

ISSN 2056-9890

Received 25 February 2016

Accepted 26 March 2016

Edited by W. T. A. Harrison, University of Aberdeen, Scotland

Keywords: crystal structure; purine- $6(9 H)$-one; 2'-deoxyguanosine; deoxy-D-ribofuranose; glycosidic linkage; nucleobase; hydrogen bonding.

CCDC reference: 1448235

Supporting information: this article has supporting information at journals.iucr.org/e

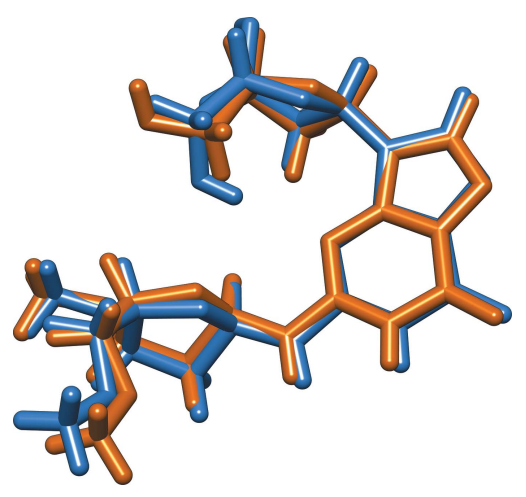

OPEN $\odot$ ACCESS

\section{Crystal structure of a nucleoside model for the interstrand cross-link formed by the reaction of 2'-deoxyguanosine and an abasic site in duplex DNA}

Michael J. Catalano, Kasi Viswanatharaju Ruddraraju, Charles L. Barnes and Kent S. Gates*

125 Chemistry Bldg, University of Missouri-Columbia, MO 65211, USA. *Correspondence e-mail: gatesk@missouri.edu

The title compound, 9-[(2R,4S,5R)-4-hydroxy-5-(hydroxymethyl)tetrahydrofuran-2-yl]-2-\{[(2R,4S,5R)-4-methoxy-5-(methoxymethyl)tetrahydrofuran-2-yl]amino\}-1H-purin-6(9H)-one, $\mathrm{C}_{17} \mathrm{H}_{25} \mathrm{~N}_{5} \mathrm{O}_{7}$, crystallizes with two independent molecules $(A$ and $B)$ in the asymmetric unit. In the crystal, the guanosine moieties of molecules $A$ and $B$ are linked by $\mathrm{N}-\mathrm{H} \cdots \mathrm{N}$ and $\mathrm{O}-\mathrm{H} \cdots \mathrm{N}$ hydrogen-bonding interactions, forming ribbons which are stacked to form columns along [100]. These columns are then linked by $\mathrm{O}-\mathrm{H} \cdots \mathrm{O}$ hydrogen bonds between the ribose moieties and numerous $\mathrm{C}-\mathrm{H} \cdots \mathrm{O}$ interactions to complete the three-dimensional structure.

\section{Chemical context}

Recent work has characterized a structurally novel set of interstrand DNA-DNA cross-links involving reaction of the ubiquitous DNA abasic lesion with a nucleobase on the opposing strand of the double helix (Catalano et al., 2015; Dutta et al., 2007; Gamboa Varela \& Gates, 2015; Johnson et al., 2013; Price et al., 2014, 2015; Yang et al., 2015; Zhang et al., 2015). Evidence indicates that the covalent attachment is forged between the anomeric carbon of the abasic sugar and the exocyclic amino group of either a guanine, adenine, or $\mathrm{N}^{4}$ aminocytosine residue (Catalano et al., 2015; Dutta et al., 2007; Gamboa Varela \& Gates, 2015; Johnson et al., 2013; Price et al., 2014, 2015; Yang et al., 2015). This type of glycosidic linkage involving the exocyclic amino group of a nucleobase is reminiscent of that found in the natural products anicemycin, spicamycin, and septacidin (Acton et al., 1977; Igarashi et al., 2005; Suzuki et al., 2002).<smiles>COC[C@H]1O[C@@H](Nc2nc3c(ncn3[C@H]3C[C@H](O)[C@@H](CO)O3)c(=O)[nH]2)C[C@@H]1OC</smiles>

Here we present single crystal X-ray crystallographic analysis of a nucleoside analog, (I), of the 2 '-deoxyguanosine/ abasic site cross-link. This structure corroborates an earlier two-dimensional NMR analysis (Catalano et al., 2015) 


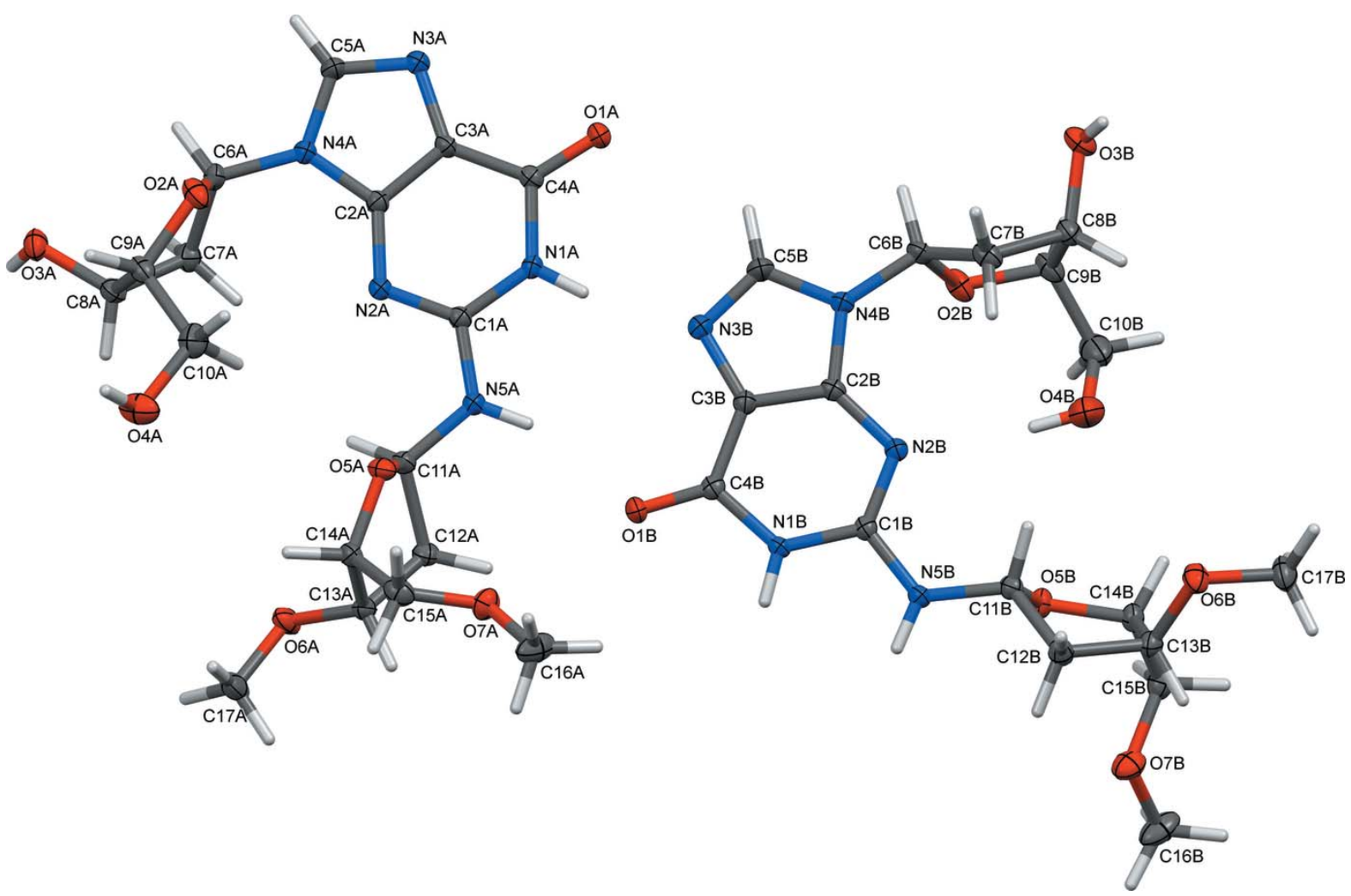

Figure 1

The molecular structure of (I) showing 50\% displacement ellipsoids.

concluding that the 2-deoxyribose unit attached at the exocyclic $N^{2}$-amino group of the guanine residue exists in the cyclic aminoglycoside form.

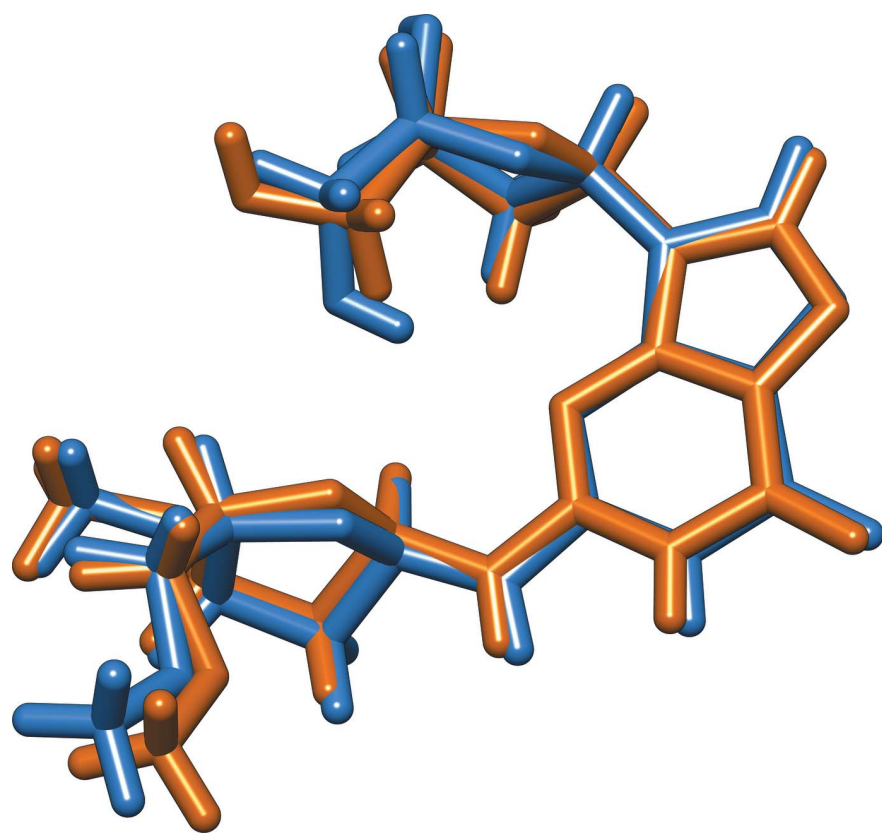

Figure 2

Overlay plot of the two molecules in (I). $A$ molecule in orange and $B$ molecule in blue.

\section{Structural commentary}

The two independent molecules ( $A$ and $B$ ) of (I) are shown in Fig. 1 as they are oriented in the crystal, while Fig. 2 shows an overlay to illustrate the differences in orientation and conformation of the furanose rings. Ring puckering analysis, after Cremer \& Pople as calculated using PLATON (Spek, 2009) indicates the furanose rings attached to N4 positions in the two molecules to be half-chairs in both molecules, but with the maximum variance from planarity occurring between $\mathrm{C} 7$ and C8 in molecule $A$ and C6 and C7 in molecule $B[Q(2)=$ $0.367(2), \Phi(2)=88.0(4)^{\circ}$ for molecule $A$ and $Q(2)=$ $0.347(2), \Phi(2)=60.6(4)^{\circ}$ for molecule $\left.B\right]$. The disposition of these furanose rings relative to the purine rings can be described by the torsion angle $\mathrm{C} 2-\mathrm{N} 4-\mathrm{C} 6-\mathrm{O} 2$, which is $70.9(3)^{\circ}$ in molecule $A$ and $61.7(3)^{\circ}$ in molecule $B$. The furanose ring attached to the N5 position in molecule $A$ is again a half-chair, with the maximum deviation from planarity between $\mathrm{C} 11 A$ and $\mathrm{C} 12 A\left[Q(2)=3.41(2), \Phi(2)=62.2(3)^{\circ}\right]$, while this furanose ring in molecule $B$ is an envelope with $\mathrm{C} 11 B$ at the flap $\left[Q(2)=0.422(2), \Phi(2)=45.4(3)^{\circ}\right]$. The disposition of these furanose rings relative to the purine rings can be described by the angle $\mathrm{C} 1-\mathrm{N} 5-\mathrm{C} 11-\mathrm{O} 5$, which is $-87.4(2)^{\circ}$ in molecule $A$ and $-93.7(2)^{\circ}$ in molecule $B$.

\section{Supramolecular features}

In the crystal, the two molecules form infinite ribbons along the $a-c$ diagonal of the unit cell, with the $A$ molecules on one 
Table 1

Hydrogen-bond geometry $\left(\AA,^{\circ}\right)$.

\begin{tabular}{lllll}
\hline$D-\mathrm{H} \cdots A$ & $D-\mathrm{H}$ & $\mathrm{H} \cdots A$ & $D \cdots A$ & $D-\mathrm{H} \cdots A$ \\
\hline $\mathrm{N} 1 A-\mathrm{H} 1 A \cdots \mathrm{N} 3 B$ & 0.88 & 1.92 & $2.789(2)$ & 170 \\
$\mathrm{O} 3 A-\mathrm{H} 3 A \cdots \mathrm{O} 5 A^{\mathrm{i}}$ & 0.84 & 2.07 & $2.897(2)$ & 167 \\
$\mathrm{O} 4 A-\mathrm{H} 4 A \cdots \mathrm{O} 3 B^{\mathrm{ii}}$ & 0.84 & 2.01 & $2.847(2)$ & 178 \\
$\mathrm{~N} 5 A-\mathrm{H} 5 A \cdots \mathrm{O} 1 B$ & 0.88 & 2.23 & $3.058(2)$ & 157 \\
$\mathrm{C} 5 A-\mathrm{H} 5 A 1 \cdots \mathrm{O} 1 B^{\mathrm{iii}}$ & 0.95 & 2.63 & $3.284(3)$ & 126 \\
$\mathrm{C} 7 A-\mathrm{H} 7 A 1 \cdots \mathrm{N} 2 A$ & 0.99 & 2.46 & $3.172(3)$ & 128 \\
$\mathrm{C} 8 A-\mathrm{H} 8 A \cdots \mathrm{O} 7 A^{\mathrm{i}}$ & 1.00 & 2.39 & $3.316(3)$ & 153 \\
$\mathrm{C} 12 A-\mathrm{H} 12 A \cdots \mathrm{O} 1 A^{\mathrm{iv}}$ & 0.99 & 2.61 & $3.432(3)$ & 141 \\
$\mathrm{C} 12 A-\mathrm{H} 12 B \cdots \mathrm{O} 1 B$ & 0.99 & 2.55 & $3.426(3)$ & 147 \\
$\mathrm{C} 16 A-\mathrm{H} 16 A \cdots \mathrm{O} 4 A^{\mathrm{v}}$ & 0.98 & 2.47 & $3.401(3)$ & 158 \\
$\mathrm{C} 16 A-\mathrm{H} 16 B \cdots \mathrm{O} 6 A^{\mathrm{v}}$ & 0.98 & 2.54 & $3.222(3)$ & 127 \\
$\mathrm{C} 16 A-\mathrm{H} 16 C \cdots \mathrm{O} 2 A^{\mathrm{vi}}$ & 0.98 & 2.50 & $3.356(3)$ & 146 \\
$\mathrm{C} 17 A-\mathrm{H} 17 A \cdots \mathrm{O} 3 A^{\mathrm{vi}}$ & 0.98 & 2.65 & $3.610(3)$ & 168 \\
$\mathrm{C} 17 A-\mathrm{H} 17 B \cdots \mathrm{O} 2 A^{\mathrm{iv}}$ & 0.98 & 2.60 & $3.573(3)$ & 175 \\
$\mathrm{~N} 1 B-\mathrm{H} 1 B \cdots \mathrm{N} 3 A^{\text {vi }}$ & 0.88 & 1.94 & $2.808(2)$ & 166 \\
$\mathrm{O} 3 B-\mathrm{H} 3 B \cdots \mathrm{O} 5 B^{\mathrm{vii}}$ & 0.84 & 1.99 & $2.817(2)$ & 169 \\
$\mathrm{O} 4 B-\mathrm{H} 4 B \cdots \mathrm{N} 2 B$ & 0.84 & 2.38 & $3.180(3)$ & 158 \\
$\mathrm{~N} 5 B-\mathrm{H} 5 B \cdots \mathrm{O} 1 A^{\mathrm{vi}}$ & 0.88 & 2.19 & $3.027(2)$ & 159 \\
$\mathrm{C} 5 B-\mathrm{H} 5 B 1 \cdots \mathrm{O} 1 A$ & 0.95 & 2.60 & $3.269(3)$ & 127 \\
$\mathrm{C} 8 B-\mathrm{H} 8 B \cdots \mathrm{O} 7 B^{\mathrm{vii}}$ & 1.00 & 2.49 & $3.363(3)$ & 146 \\
$\mathrm{C} 11 B-\mathrm{H} 11 B \cdots \mathrm{O} 4 B$ & 1.00 & 2.59 & $3.251(3)$ & 124 \\
$\mathrm{C} 12 B-\mathrm{H} 12 C \cdots \mathrm{O} 1 A^{\text {vi }}$ & 0.99 & 2.55 & $3.363(3)$ & 140 \\
$\mathrm{C} 12 B-\mathrm{H} 12 D \cdots \mathrm{O} 1 B^{\mathrm{v}}$ & 0.99 & 2.45 & $3.424(3)$ & 167 \\
$\mathrm{C} 14 B-\mathrm{H} 14 B \cdots \mathrm{O} 4 B$ & 1.00 & 2.61 & $3.272(3)$ & 123 \\
$\mathrm{C} 17 B-\mathrm{H} 17 E \cdots \mathrm{O} 2 B^{\mathrm{v}}$ & 0.98 & 2.48 & $3.456(3)$ & 176 \\
\hline
\end{tabular}

Symmetry codes: (i) $x-1, y, z$; (ii) $-x+1, y+\frac{1}{2},-z$; (iii) $x-1, y, z-1$; (iv) $x, y, z+1$; (v) $x+1, y, z ;$ (vi) $x+1, y, z+1$; (vii) $x, y, z-1$.

side of the ribbon and the $B$ molecules on the other. The molecules are staggered such that each $A$ molecule forms hydrogen bonds to two $B$ molecules and each $B$ molecule forms hydrogen bonds (Table 1) to two $A$ molecules, fully involving the N1, N3, N5 and O1 atoms. These ribbons are then stacked to form slabs propagating in the $a c$ plane and one half the $b$ dimension in thickness. The deoxyribose moieties occupy the outsides of these slabs and are linked via hydrogen bonds to twofold screw-related slabs, resulting in a herringbone pattern in the three-dimensional structure as seen in Fig. 3.

\section{Database survey}

A search of the Cambridge Structural Database (CSD, Version 5.36, update February 2015; Groom \& Allen, 2014) for deoxyguanosine analogues with exocyclic amine substitution revealed three crystal structures (Morr et al., 1991; Fujino et al., 2010). In all these crystal structures, the five-membered 2deoxyribofuranose rings have envelope conformations, as in the title compound.

\section{Synthesis and crystallization}

2'-Deoxyguanosine $(199 \mathrm{mg}, \quad 0.75 \mathrm{mmol})$ and 3,5-bis- $O$ methyl-2-deoxy-D-ribofuranose $(110 \mathrm{mg}, 0.74 \mathrm{mmol}$ ) were dissolved in $0.8 \mathrm{ml}$ of a 3:1 mixture of DMSO and $25 \mathrm{mM}$ sodium phosphate buffer $(\mathrm{pH} 7.0)$ in a round-bottom flask. The flask was heated to $333 \mathrm{~K}$ and the mixture stirred for $22 \mathrm{~h}$. The solvent removed in vacuo and the product purified by column chromatography on silica gel eluted with $0-15 \%$ methanol in dichloromethane $\left(R_{f}=0.30,15 \%\right.$ methanol/di-

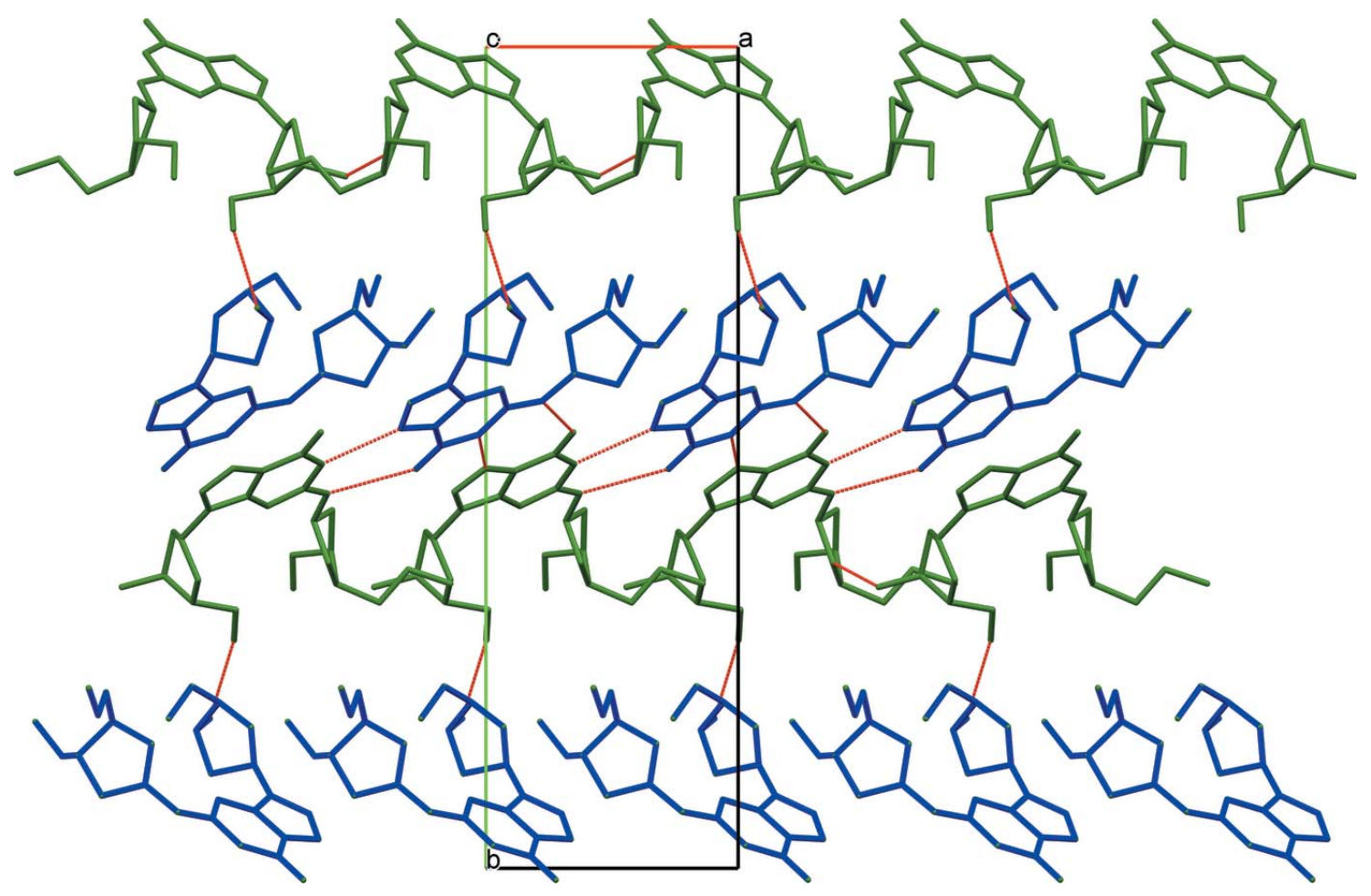

Figure 3

The packing in (I) along the $c$ axis showing the formation of hydrogen-bonded chains ( $A$ molecules green, $B$ molecules blue). 
chloromethane) to yield $36 \mathrm{mg}$ ( $12 \%$ yield) of the title compound as a colorless oil. The precursor 3,5-bis- $O$-methyl2-deoxy-D-ribofuranose was synthesized according to previously reported procedures (Deriaz et al., 1949; Olsson et al., 1998). The title compound was crystallized by vapour diffusion, a $2 \mathrm{ml}$ vial containing the title compound in methanol being placed in a $20 \mathrm{ml}$ vial containing hexanes at room temperature for several days.

\section{Refinement}

Crystal data, data collection and structure refinement details are summarized in Table 2 . $\mathrm{H}$ atoms were placed geometrically $(\mathrm{C}-\mathrm{H}=0.95$ or $0.98 \AA)$ and refined as riding with $U_{\text {iso }}(\mathrm{H})=$ $1.2 U_{\text {eq }}(\mathrm{C})$.

\section{References}

Acton, E. M., Ryan, K. J. \& Luetzow, A. E. (1977). J. Med. Chem. 20, 1362-1371.

Barbour, L. J. (2001). J. Supramol. Chem. 1, 189-191.

Bruker (2008). APEX2, SAINT and SADABS. Bruker AXS Inc., Madison, Wisconsin, USA.

Catalano, M. J., Liu, S., Andersen, N., Yang, Z., Johnson, K. M., Price, N. A., Wang, Y. \& Gates, K. S. (2015). J. Am. Chem. Soc. 137, 39333945.

Deriaz, R. E., Overend, W. G., Stacey, M. \& Wiggins, L. F. (1949). J. Chem. Soc. pp. 2836-2841.

Dutta, S., Chowdhury, G. \& Gates, K. S. (2007). J. Am. Chem. Soc. 129, 1852-1853.

Fujino, T., Tsunaka, N., Guillot-Nieckowski, M., Nakanishi, W., Iwamoto, T., Nakamura, E. \& Isobe, H. (2010). Tetrahedron Lett. 51, 2036-2038.

Gamboa Varela, J. \& Gates, K. S. (2015). Angew. Chem. Int. Ed. 54, 7666-7669.

Groom, C. R. \& Allen, F. H. (2014). Angew. Chem. Int. Ed. 53, 662671.

Igarashi, Y., Ootsu, K., Onaka, H., Fujita, T., Uehara, Y. \& Furumai, T. (2005). J. Antibiot. 58, 322-326.

Johnson, K. M., Price, N. E., Wang, J., Fekry, M. I., Dutta, S., Seiner, D. R., Wang, Y. \& Gates, K. S. (2013). J. Am. Chem. Soc. 135, 10151025.

Morr, M., Ernst, L. \& Schomburg, D. (1991). Liebigs Ann. Chem. 1991, 615-631.

Olsson, R., Rundström, P. \& Frejd, T. (1998). J. Chem. Soc. Perkin Trans. 1, pp. 785-790.

Parsons, S., Flack, H. D. \& Wagner, T. (2013). Acta Cryst. B69, 249259.
Table 2

Experimental details.

\begin{tabular}{ll}
\hline Crystal data & \\
Chemical formula & $\mathrm{C}_{17} \mathrm{H}_{25} \mathrm{~N}_{5} \mathrm{O}_{7}$ \\
$M_{\mathrm{r}}$ & 411.42 \\
Crystal system, space group & Monoclinic, $P 2_{1}$ \\
Temperature $(\mathrm{K})$ & 100 \\
$a, b, c(\AA)$ & $8.1817(1), 26.4033(5), 8.8800(2)$ \\
$\beta\left({ }^{\circ}\right.$ & $98.023(1)$ \\
$V\left(\AA^{3}\right)$ & $1899.52(6)$ \\
$Z$ & 4 \\
Radiation type & $\mathrm{Cu} \mathrm{K \alpha}$ \\
$\mu\left(\mathrm{mm}^{-1}\right)$ & 0.96 \\
Crystal size $(\mathrm{mm})$ & $0.15 \times 0.08 \times 0.08$ \\
& \\
Data collection & Bruker APEXII CCD \\
Diffractometer & Multi-scan $(S A D A B S ;$ Sheldrick, \\
Absorption correction & $2008)$ \\
& $0.86,0.93$ \\
$T_{\text {min }}, T_{\text {max }}$ & $26696,6862,6644$ \\
No. of measured, independent and & \\
observed $[I>2 \sigma(I)]$ reflections & 0.029 \\
$R_{\text {int }}$ & 0.617 \\
(sin $\theta / \lambda)_{\text {max }}\left(\AA^{-1}\right)$ & \\
Refinement & \\
$R\left[F^{2}>2 \sigma\left(F^{2}\right)\right], w R\left(F^{2}\right), S$ & $0.027,0.072,1.04$ \\
No. of reflections & 6862 \\
No. of parameters & 531 \\
No. of restraints & 1 \\
$H$-atom treatment & H-atom parameters constrained \\
$\Delta \rho_{\text {max }}, \Delta \rho_{\text {min }}\left(\mathrm{e} \AA^{-3}\right)$ & $0.23,-0.17$ \\
Absolute structure & Flack $x$ determined using 2923 \\
& quotients $\left[\left(I^{+}\right)-\left(I^{-}\right)\right] /\left[\left(I^{+}\right)+\left(I^{-}\right)\right]$ \\
Absolute structure parameter & $0.08(5)$ \\
\hline
\end{tabular}

Computer programs: APEX2 (Bruker, 2008), SAINT (Bruker, 2008), SHELXS2013 (Sheldrick, 2008), SHELXL2013 (Sheldrick, 2015), X-SEED, Barbour, 2001, CIFTAB (Sheldrick, 2008).

Price, N. E., Catalano, M. J., Liu, S., Wang, Y. \& Gates, K. S. (2015). Nucleic Acids Res. 43, 3434-3441.

Price, N. E., Johnson, K. M., Wang, J., Fekry, M. I., Wang, Y. \& Gates, K. S. (2014). J. Am. Chem. Soc. 136, 3483-3490.

Sheldrick, G. M. (2008). Acta Cryst. A64, 112-122.

Sheldrick, G. M. (2015). Acta Cryst. C71, 3-8.

Spek, A. L. (2009). Acta Cryst. D65, 148-155.

Suzuki, T., Suzuki, S. T., Yamada, I., Koashi, Y., Yamada, K. \& Chida, N. (2002). J. Org. Chem. 67, 2874-2880.

Yang, Z., Price, N. E., Johnson, K. M. \& Gates, K. S. (2015). Biochemistry, 54, 4259-4266.

Zhang, X., Price, N. E., Fang, X., Yang, Z., Gu, L. Q. \& Gates, K. S. (2015). ACS Nano, 9, 11812-11819. 


\section{supporting information}

Acta Cryst. (2016). E72, 624-627 [doi:10.1107/S205698901600517X]

Crystal structure of a nucleoside model for the interstrand cross-link formed by the reaction of 2'-deoxyguanosine and an abasic site in duplex DNA

Michael J. Catalano, Kasi Viswanatharaju Ruddraraju, Charles L. Barnes and Kent S. Gates

Computing details

Data collection: APEX2 (Bruker, 2008); cell refinement: SAINT (Bruker, 2008); data reduction: SAINT (Bruker, 2008); program(s) used to solve structure: SHELXS2013 (Sheldrick, 2008); program(s) used to refine structure: SHELXL2013 (Sheldrick, 2015); molecular graphics: X-SEED, Barbour, 2001; software used to prepare material for publication: CIFTAB (Sheldrick, 2008).

9-[(2R,4S,5R)-4-Hydroxy-5-(hydroxymethyl)tetrahydrofuran-2-yl]-2-\{[(2R,4S,5R)-4methoxy-5-(methoxymethyl)tetrahydrofuran-2-yl]amino\}-1H-purin-6(9H)-one

Crystal data

$\mathrm{C}_{17} \mathrm{H}_{25} \mathrm{~N}_{5} \mathrm{O}_{7}$

$M_{r}=411.42$

Monoclinic, $P 2_{1}$ $a=8.1817(1) \AA$

$b=26.4033(5) \AA$

$c=8.8800(2) \AA$

$\beta=98.023(1)^{\circ}$

$V=1899.52(6) \AA^{3}$

$Z=4$

Data collection

Bruker APEXII CCD

diffractometer

Radiation source: Incoatec micro focus $\mathrm{Cu}$ tube $\omega$ and phi scans

Absorption correction: multi-scan

(SADABS; Sheldrick, 2008)

$T_{\min }=0.86, T_{\max }=0.93$

26696 measured reflections

Refinement

Refinement on $F^{2}$

Least-squares matrix: full

$R\left[F^{2}>2 \sigma\left(F^{2}\right)\right]=0.027$

$w R\left(F^{2}\right)=0.072$

$S=1.04$

6862 reflections

531 parameters

1 restraint
$F(000)=872$

$D_{\mathrm{x}}=1.439 \mathrm{Mg} \mathrm{m}^{-3}$

$\mathrm{Cu} K \alpha$ radiation, $\lambda=1.54178 \AA$

Cell parameters from 9940 reflections

$\theta=5.3-72.2^{\circ}$

$\mu=0.96 \mathrm{~mm}^{-1}$

$T=100 \mathrm{~K}$

Prism, colourless

$0.15 \times 0.08 \times 0.08 \mathrm{~mm}$

6862 independent reflections

6644 reflections with $I>2 \sigma(I)$

$R_{\text {int }}=0.029$

$\theta_{\max }=72.2^{\circ}, \theta_{\min }=3.4^{\circ}$

$h=-10 \rightarrow 10$

$k=-31 \rightarrow 31$

$l=-9 \rightarrow 10$

Hydrogen site location: inferred from neighbouring sites

$\mathrm{H}$-atom parameters constrained

$w=1 /\left[\sigma^{2}\left(F_{\mathrm{o}}^{2}\right)+(0.0424 P)^{2}+0.3476 P\right]$

where $P=\left(F_{\mathrm{o}}^{2}+2 F_{\mathrm{c}}{ }^{2}\right) / 3$

$(\Delta / \sigma)_{\max }<0.001$

$\Delta \rho_{\max }=0.23 \mathrm{e} \AA^{-3}$

$\Delta \rho_{\min }=-0.17 \mathrm{e}^{-3}$ 
Absolute structure: Flack $x$ determined using 2923 quotients $\left[\left(I^{+}\right)-\left(I^{-}\right)\right] /\left[\left(I^{+}\right)+\left(I^{-}\right)\right]$(Parsons et al., 2013)

Absolute structure parameter: 0.08 (5)

\section{Special details}

Geometry. All esds (except the esd in the dihedral angle between two 1.s. planes) are estimated using the full covariance matrix. The cell esds are taken into account individually in the estimation of esds in distances, angles and torsion angles; correlations between esds in cell parameters are only used when they are defined by crystal symmetry. An approximate (isotropic) treatment of cell esds is used for estimating esds involving l.s. planes.

Fractional atomic coordinates and isotropic or equivalent isotropic displacement parameters $\left(\AA^{2}\right)$

\begin{tabular}{|c|c|c|c|c|}
\hline & $x$ & $y$ & $z$ & $U_{\text {iso }} * / U_{\text {eq }}$ \\
\hline O1A & $0.34576(17)$ & $0.46915(6)$ & $-0.12995(17)$ & $0.0168(3)$ \\
\hline N1A & $0.3499(2)$ & $0.50765(6)$ & $0.1022(2)$ & $0.0146(3)$ \\
\hline H1A & 0.4493 & 0.4955 & 0.1317 & $0.017^{*}$ \\
\hline $\mathrm{C} 1 \mathrm{~A}$ & $0.2806(2)$ & $0.53738(8)$ & $0.2030(2)$ & $0.0140(4)$ \\
\hline $\mathrm{O} 2 \mathrm{~A}$ & $-0.1409(2)$ & $0.64849(6)$ & $0.01984(18)$ & $0.0223(3)$ \\
\hline $\mathrm{N} 2 \mathrm{~A}$ & $0.1339(2)$ & $0.55894(7)$ & $0.1729(2)$ & $0.0155(4)$ \\
\hline $\mathrm{C} 2 \mathrm{~A}$ & $0.0560(3)$ & $0.54690(8)$ & $0.0330(2)$ & $0.0150(4)$ \\
\hline $\mathrm{O} 3 \mathrm{~A}$ & $-0.44313(19)$ & $0.65756(6)$ & $0.18573(19)$ & $0.0221(3)$ \\
\hline $\mathrm{H} 3 \mathrm{~A}$ & -0.5051 & 0.6460 & 0.2454 & $0.027^{*}$ \\
\hline N3A & $-0.0017(2)$ & $0.51309(7)$ & $-0.2041(2)$ & $0.0170(4)$ \\
\hline $\mathrm{C} 3 \mathrm{~A}$ & $0.1129(2)$ & $0.51624(8)$ & $-0.0744(2)$ & $0.0148(4)$ \\
\hline $\mathrm{O} 4 \mathrm{~A}$ & $0.0015(2)$ & $0.72195(6)$ & $0.3635(2)$ & $0.0298(4)$ \\
\hline $\mathrm{H} 4 \mathrm{~A}$ & -0.0253 & 0.7501 & 0.3235 & $0.036^{*}$ \\
\hline $\mathrm{N} 4 \mathrm{~A}$ & $-0.0978(2)$ & $0.56295(7)$ & $-0.0324(2)$ & $0.0177(4)$ \\
\hline $\mathrm{C} 4 \mathrm{~A}$ & $0.2740(2)$ & $0.49535(7)$ & $-0.0439(2)$ & $0.0138(4)$ \\
\hline O5A & $0.37552(18)$ & $0.62783(6)$ & $0.42933(16)$ & $0.0176(3)$ \\
\hline N5A & $0.3711(2)$ & $0.54372(7)$ & $0.3418(2)$ & 0.0163 (4) \\
\hline $\mathrm{H} 5 \mathrm{~A}$ & 0.4647 & 0.5272 & 0.3642 & $0.020^{*}$ \\
\hline $\mathrm{C} 5 \mathrm{~A}$ & $-0.1246(3)$ & $0.54152(8)$ & $-0.1750(2)$ & $0.0192(4)$ \\
\hline H5A1 & -0.2220 & 0.5468 & -0.2449 & $0.023^{*}$ \\
\hline O6A & $0.23353(18)$ & $0.61868(6)$ & $0.75564(18)$ & $0.0227(3)$ \\
\hline C6A & $-0.2089(3)$ & $0.59897(8)$ & $0.0258(3)$ & $0.0185(4)$ \\
\hline H6A & -0.3179 & 0.5980 & -0.0410 & $0.022^{*}$ \\
\hline O7A & $0.69231(18)$ & $0.63615(6)$ & $0.60640(19)$ & $0.0221(3)$ \\
\hline C7A & $-0.2373(3)$ & $0.59060(8)$ & $0.1890(3)$ & $0.0184(4)$ \\
\hline H7A1 & -0.1372 & 0.5770 & 0.2514 & $0.022^{*}$ \\
\hline H7A2 & -0.3309 & 0.5673 & 0.1948 & $0.022^{*}$ \\
\hline $\mathrm{C} 8 \mathrm{~A}$ & -0.2764 & $0.64379(8)$ & $0.2388(2)$ & $0.0182(4)$ \\
\hline $\mathrm{H} 8 \mathrm{~A}$ & -0.2507 & 0.6476 & 0.3516 & $0.022^{*}$ \\
\hline C9A & $-0.1612(3)$ & $0.67599(8)$ & $0.1574(2)$ & $0.0189(4)$ \\
\hline H9A & -0.2152 & 0.7093 & 0.1293 & $0.023^{*}$ \\
\hline $\mathrm{C} 10 \mathrm{~A}$ & $0.0085(3)$ & $0.68527(9)$ & $0.2482(3)$ & $0.0245(5)$ \\
\hline $\mathrm{H} 10 \mathrm{~A}$ & 0.0518 & 0.6531 & 0.2951 & $0.029^{*}$ \\
\hline H10B & 0.0853 & 0.6970 & 0.1787 & $0.029^{*}$ \\
\hline C11A & $0.3171(2)$ & $0.57690(8)$ & $0.4527(2)$ & $0.0156(4)$ \\
\hline
\end{tabular}




\begin{tabular}{|c|c|c|c|c|}
\hline H11A & 0.1938 & 0.5767 & 0.4429 & $0.019^{*}$ \\
\hline C12A & $0.3901(3)$ & $0.56396(8)$ & $0.6145(2)$ & $0.0182(4)$ \\
\hline $\mathrm{H} 12 \mathrm{~A}$ & 0.3228 & 0.5381 & 0.6581 & $0.022 *$ \\
\hline H12B & 0.5045 & 0.5513 & 0.6190 & $0.022 *$ \\
\hline C13A & $0.3861(2)$ & $0.61397(8)$ & 0.6978 (2) & $0.0167(4)$ \\
\hline H13A & 0.4804 & 0.6161 & 0.7823 & $0.020 *$ \\
\hline $\mathrm{C} 14 \mathrm{~A}$ & $0.4064(3)$ & $0.65359(8)$ & $0.5738(2)$ & $0.0175(4)$ \\
\hline H14A & 0.3230 & 0.6811 & 0.5769 & $0.021 *$ \\
\hline $\mathrm{C} 15 \mathrm{~A}$ & $0.5775(3)$ & $0.67638(9)$ & $0.5938(3)$ & 0.0218 \\
\hline H15A & 0.5917 & 0.6979 & 0.5053 & $0.026^{*}$ \\
\hline H15B & 0.5947 & 0.6976 & 0.6866 & $0.026^{*}$ \\
\hline $\mathrm{C} 16 \mathrm{~A}$ & $0.8590(3)$ & $0.65335(11)$ & $0.6422(3)$ & $0.0301(5)$ \\
\hline $\mathrm{H} 16 \mathrm{~A}$ & 0.8799 & 0.6802 & 0.5711 & $0.045^{*}$ \\
\hline H16B & 0.9346 & 0.6250 & 0.6338 & $0.045^{*}$ \\
\hline H16C & 0.8766 & 0.6666 & 0.7463 & $0.045^{*}$ \\
\hline C17A & $0.2364(3)$ & $0.65893(11)$ & $0.8622(3)$ & $0.0289(5)$ \\
\hline H17A & 0.3324 & 0.6551 & 0.9407 & $0.043^{*}$ \\
\hline H17B & 0.1352 & 0.6581 & 0.9096 & $0.043^{*}$ \\
\hline $\mathrm{H} 17 \mathrm{C}$ & 0.2435 & 0.6913 & 0.8097 & $0.043 *$ \\
\hline O1B & $0.72354(17)$ & $0.51384(5)$ & 0.47267 (17) & $0.0168(3)$ \\
\hline N1B & $0.9716(2)$ & $0.47201(6)$ & $0.50184(19)$ & $0.0139(3)$ \\
\hline H1B & 0.9971 & 0.4855 & 0.5927 & $0.017^{*}$ \\
\hline $\mathrm{C} 1 \mathrm{~B}$ & $1.0833(2)$ & $0.43911(7)$ & $0.4530(2)$ & $0.0136(4)$ \\
\hline $\mathrm{O} 2 \mathrm{~B}$ & $0.9310(2)$ & $0.32766(6)$ & $0.07593(19)$ & $0.0219(3)$ \\
\hline $\mathrm{N} 2 \mathrm{~B}$ & $1.0610(2)$ & $0.41620(6)$ & $0.3193(2)$ & $0.0153(4)$ \\
\hline $\mathrm{C} 2 \mathrm{~B}$ & 0.9168 (3) & $0.42949(8)$ & $0.2340(2)$ & $0.0150(4)$ \\
\hline $\mathrm{O} 3 \mathrm{~B}$ & $1.09413(19)$ & $0.31769(6)$ & $-0.23244(18)$ & $0.0220(3)$ \\
\hline $\mathrm{H} 3 \mathrm{~B}$ & 1.1571 & 0.3306 & -0.2890 & $0.026^{*}$ \\
\hline N3B & $0.6634(2)$ & $0.46447(7)$ & $0.1606(2)$ & $0.0188(4)$ \\
\hline $\mathrm{C} 3 \mathrm{~B}$ & $0.7979(2)$ & $0.46230(8)$ & $0.2729(2)$ & $0.0159(4)$ \\
\hline O4B & $1.2568(3)$ & $0.31747(9)$ & $0.2436(2)$ & $0.0452(5)$ \\
\hline $\mathrm{H} 4 \mathrm{~B}$ & 1.1920 & 0.3377 & 0.2779 & $0.054^{*}$ \\
\hline N4B & $0.8528(2)$ & $0.41146(7)$ & $0.0918(2)$ & $0.0186(4)$ \\
\hline C4B & $0.8210(2)$ & $0.48559(8)$ & $0.4184(2)$ & $0.0143(4)$ \\
\hline $\mathrm{O} 5 \mathrm{~B}$ & $1.32491(17)$ & $0.34807(6)$ & $0.57774(18)$ & 0.0175 \\
\hline N5B & $1.2221(2)$ & $0.43116(7)$ & $0.5529(2)$ & $0.0159(3)$ \\
\hline H5B & 1.2334 & 0.4468 & 0.6413 & $0.019^{*}$ \\
\hline C5B & $0.7002(3)$ & $0.43335(9)$ & $0.0558(3)$ & $0.0215(5)$ \\
\hline H5B1 & 0.6287 & 0.4266 & -0.0358 & $0.026^{*}$ \\
\hline O6B & 1.67925 (19) & $0.36348(6)$ & $0.43876(18)$ & $0.0216(3)$ \\
\hline $\mathrm{C} 6 \mathrm{~B}$ & 0.9187 (3) & $0.37405(8)$ & $-0.0034(2)$ & $0.0185(4)$ \\
\hline H6B & 0.8402 & 0.3699 & -0.0995 & $0.022 *$ \\
\hline O7B & $1.5367(2)$ & $0.31343(6)$ & 0.84948 (19) & $0.0252(3)$ \\
\hline C7B & $1.0893(3)$ & $0.38469(8)$ & $-0.0431(3)$ & $0.0198(4)$ \\
\hline H7B1 & 1.1615 & 0.4002 & 0.0435 & $0.024 *$ \\
\hline H7B2 & 1.0845 & 0.4072 & -0.1328 & $0.024 *$ \\
\hline $\mathrm{C} 8 \mathrm{~B}$ & $1.1484(3)$ & $0.33176(8)$ & $-0.0781(2)$ & $0.0191(4)$ \\
\hline H8B & 1.2709 & 0.3287 & -0.0525 & $0.023 *$ \\
\hline
\end{tabular}




$\begin{array}{lllll}\text { C9B } & 1.0581(3) & 0.29760(9) & 0.0248(3) & 0.0226(5) \\ \text { H9B } & 1.0071 & 0.2683 & -0.0357 & 0.07^{*} \\ \text { C10B } & 1.1657(4) & 0.27835(11) & 0.1641(3) & 0.0360(6) \\ \text { H10C } & 1.0958 & 0.2618 & 0.2322 & 0.043^{*} \\ \text { H10D } & 1.2427 & 0.2526 & 0.1337 & 0.043^{*} \\ \text { C11B } & 1.3507(3) & 0.39806(8) & 0.5188(2) & 0.0157(4) \\ \text { H11B } & 1.3500 & 0.3963 & 0.4062 & 0.019^{*} \\ \text { C12B } & 1.5222(3) & 0.41144(8) & 0.5959(3) & 0.0191(4) \\ \text { H12C } & 1.5218 & 0.4204 & 0.7041 & 0.023^{*} \\ \text { H12D } & 1.5700 & 0.4398 & 0.5436 & 0.023^{*} \\ \text { C13B } & 1.6149(3) & 0.36211(8) & 0.5792(2) & 0.0177(4) \\ \text { H13B } & 1.7050 & 0.3572 & 0.6664 & 0.021^{*} \\ \text { C14B } & 1.4792(3) & 0.32117(8) & 0.5795(3) & 0.0178(4) \\ \text { H14B } & 1.4738 & 0.3009 & 0.4838 & 0.021^{*} \\ \text { C15B } & 1.5051(3) & 0.28550(9) & 0.7128(3) & 0.0227(5) \\ \text { H15C } & 1.4055 & 0.2643 & 0.7142 & 0.027^{*} \\ \text { H15D } & 1.5995 & 0.2628 & 0.7033 & 0.027^{*} \\ \text { C16B } & 1.5735(3) & 0.28046(11) & 0.9765(3) & 0.0331^{*}(6) \\ \text { H16D } & 1.4856 & 0.2552 & 0.9752 & 0.050^{*} \\ \text { H16E } & 1.5814 & 0.3002 & 1.0708 & 0.050^{*} \\ \text { H16F } & 1.6788 & 0.2633 & 0.9711 & 0.050^{*} \\ \text { C17B } & 1.7854(3) & 0.32160(10) & 0.4226(3) & 0.0271^{(5)} \\ \text { H17D } & 1.8756 & 0.3212 & 0.5077 & 0.041^{*} \\ \text { H17E } & 1.8312 & 0.3248 & 0.3267 & 0.041^{*} \\ \text { H17F } & 1.7225 & 0.2900 & 0.4221 & 0.041^{*} \\ & & & & \end{array}$

Atomic displacement parameters $\left(\AA^{2}\right)$

\begin{tabular}{lllllll}
\hline & $U^{11}$ & $U^{22}$ & $U^{33}$ & $U^{12}$ & $U^{13}$ & $U^{23}$ \\
\hline O1A & $0.0171(7)$ & $0.0182(7)$ & $0.0155(7)$ & $0.0035(6)$ & $0.0033(5)$ & $-0.0025(6)$ \\
N1A & $0.0120(7)$ & $0.0159(8)$ & $0.0155(9)$ & $0.0028(6)$ & $0.0006(6)$ & $-0.0015(6)$ \\
C1A & $0.0148(9)$ & $0.0128(9)$ & $0.0142(10)$ & $-0.0002(7)$ & $0.0018(7)$ & $-0.0013(7)$ \\
O2A & $0.0304(9)$ & $0.0195(8)$ & $0.0191(8)$ & $0.0039(6)$ & $0.0108(6)$ & $0.0007(6)$ \\
N2A & $0.0152(8)$ & $0.0175(9)$ & $0.0134(9)$ & $0.0031(6)$ & $0.0008(7)$ & $-0.0026(7)$ \\
C2A & $0.0152(9)$ & $0.0136(10)$ & $0.0158(10)$ & $0.0012(7)$ & $0.0005(7)$ & $-0.0019(7)$ \\
O3A & $0.0198(8)$ & $0.0247(8)$ & $0.0234(8)$ & $0.0066(6)$ & $0.0078(6)$ & $0.0027(6)$ \\
N3A & $0.0174(8)$ & $0.0183(9)$ & $0.0148(9)$ & $0.0033(7)$ & $0.0001(7)$ & $-0.0032(7)$ \\
C3A & $0.0170(9)$ & $0.0137(9)$ & $0.0134(10)$ & $0.0018(8)$ & $0.0014(8)$ & $-0.0032(7)$ \\
O4A & $0.0448(10)$ & $0.0194(8)$ & $0.0243(9)$ & $-0.0047(7)$ & $0.0011(7)$ & $0.0001(7)$ \\
N4A & $0.0172(8)$ & $0.0195(9)$ & $0.0154(9)$ & $0.0053(7)$ & $-0.0012(7)$ & $-0.0036(7)$ \\
C4A & $0.0152(10)$ & $0.0127(10)$ & $0.0139(10)$ & $-0.0012(7)$ & $0.0032(8)$ & $-0.0005(7)$ \\
O5A & $0.0227(7)$ & $0.0174(7)$ & $0.0123(7)$ & $-0.0008(6)$ & $0.0009(5)$ & $-0.0013(6)$ \\
N5A & $0.0139(8)$ & $0.0201(9)$ & $0.0142(9)$ & $0.0039(6)$ & $-0.0003(6)$ & $-0.0034(7)$ \\
C5A & $0.0169(10)$ & $0.0228(11)$ & $0.0166(11)$ & $0.0045(8)$ & $-0.0027(8)$ & $-0.0048(8)$ \\
O6A & $0.0163(7)$ & $0.0333(9)$ & $0.0194(8)$ & $-0.0025(6)$ & $0.0060(6)$ & $-0.0082(7)$ \\
C6A & $0.0163(9)$ & $0.0200(11)$ & $0.0185(11)$ & $0.0069(8)$ & $0.0005(8)$ & $-0.0024(8)$ \\
O7A & $0.0153(7)$ & $0.0240(8)$ & $0.0268(9)$ & $-0.0015(6)$ & $0.0027(6)$ & $0.0011(6)$ \\
C7A & $0.0172(10)$ & $0.0172(11)$ & $0.0211(11)$ & $0.0001(8)$ & $0.0037(8)$ & $0.0002(8)$
\end{tabular}




\begin{tabular}{|c|c|c|c|c|c|c|}
\hline C8A & $0.0203(10)$ & $0.0201(11)$ & $0.0148(10)$ & $0.0015(8)$ & $0.0047(8)$ & $-0.0008(8)$ \\
\hline C9A & $0.0257(11)$ & $0.0157(10)$ & $0.0165(10)$ & $0.0038(8)$ & $0.0073(8)$ & $0.0009(8)$ \\
\hline $\mathrm{C} 10 \mathrm{~A}$ & $0.0259(12)$ & $0.0182(11)$ & $0.0297(13)$ & $0.0001(9)$ & $0.0052(10)$ & $0.0023(9)$ \\
\hline C11A & $0.0150(9)$ & $0.0167(10)$ & $0.0149(10)$ & $0.0005(7)$ & $0.0015(7)$ & $-0.0028(8)$ \\
\hline $\mathrm{C} 12 \mathrm{~A}$ & $0.0206(10)$ & $0.0201(10)$ & $0.0137(10)$ & $0.0017(8)$ & $0.0024(8)$ & $-0.0002(8)$ \\
\hline C13A & $0.0139(9)$ & $0.0227(11)$ & $0.0134(10)$ & $0.0007(8)$ & $0.0012(7)$ & $-0.0029(8)$ \\
\hline C14A & $0.0185(10)$ & $0.0172(10)$ & $0.0163(10)$ & $0.0037(8)$ & $0.0004(8)$ & $-0.0042(8)$ \\
\hline $\mathrm{C} 15 \mathrm{~A}$ & $0.0257(11)$ & $0.0193(11)$ & $0.0207(11)$ & $-0.0025(9)$ & $0.0043(9)$ & $-0.0023(9)$ \\
\hline $\mathrm{C} 16 \mathrm{~A}$ & $0.0209(11)$ & $0.0458(15)$ & $0.0230(12)$ & $-0.0085(10)$ & $0.0010(9)$ & $0.0059(11)$ \\
\hline C17A & $0.0229(11)$ & $0.0410(14)$ & $0.0238(12)$ & $0.0011(10)$ & $0.0065(9)$ & $-0.0138(10)$ \\
\hline O1B & $0.0172(7)$ & $0.0172(7)$ & $0.0163(7)$ & $0.0052(6)$ & $0.0031(6)$ & $-0.0026(6)$ \\
\hline N1B & $0.0149(8)$ & $0.0147(8)$ & $0.0119(8)$ & $0.0022(6)$ & $0.0009(6)$ & $-0.0038(6)$ \\
\hline $\mathrm{C} 1 \mathrm{~B}$ & $0.0148(9)$ & $0.0122(9)$ & $0.0140(10)$ & $0.0001(7)$ & $0.0033(7)$ & $0.0005(7)$ \\
\hline $\mathrm{O} 2 \mathrm{~B}$ & $0.0261(8)$ & $0.0176(8)$ & $0.0243(8)$ & $-0.0016(6)$ & $0.0113(6)$ & $-0.0048(6)$ \\
\hline $\mathrm{N} 2 \mathrm{~B}$ & $0.0137(8)$ & $0.0171(9)$ & $0.0148(9)$ & $0.0019(6)$ & $0.0010(6)$ & $-0.0028(7)$ \\
\hline $\mathrm{C} 2 \mathrm{~B}$ & $0.0169(9)$ & $0.0146(10)$ & $0.0136(10)$ & $-0.0007(8)$ & $0.0022(7)$ & $-0.0021(8)$ \\
\hline O3B & $0.0278(8)$ & $0.0215(8)$ & $0.0183(8)$ & $-0.0035(6)$ & $0.0094(6)$ & $-0.0033(6)$ \\
\hline N3B & $0.0168(8)$ & $0.0222(9)$ & $0.0166(9)$ & $0.0042(7)$ & $-0.0003(7)$ & $-0.0038(7)$ \\
\hline $\mathrm{C} 3 \mathrm{~B}$ & $0.0144(9)$ & $0.0162(10)$ & $0.0168(10)$ & $0.0017(8)$ & $0.0014(8)$ & $-0.0016(8)$ \\
\hline $\mathrm{O} 4 \mathrm{~B}$ & $0.0386(11)$ & $0.0665(15)$ & $0.0278(10)$ & $0.0170(10)$ & $-0.0056(8)$ & $-0.0101(10)$ \\
\hline N4B & $0.0168(9)$ & $0.0227(9)$ & $0.0153(9)$ & $0.0048(7)$ & $-0.0013(7)$ & $-0.0068(7)$ \\
\hline C4B & $0.0154(9)$ & $0.0128(9)$ & $0.0147(10)$ & $-0.0004(7)$ & $0.0024(7)$ & $0.0014(7)$ \\
\hline $\mathrm{O} 5 \mathrm{~B}$ & $0.0139(7)$ & $0.0165(7)$ & $0.0226(8)$ & $0.0023(6)$ & $0.0037(6)$ & $0.0013(6)$ \\
\hline N5B & $0.0172(8)$ & $0.0162(8)$ & $0.0137(8)$ & $0.0038(7)$ & $0.0004(6)$ & $-0.0042(6)$ \\
\hline $\mathrm{C} 5 \mathrm{~B}$ & $0.0185(10)$ & $0.0270(12)$ & $0.0174(11)$ & $0.0039(9)$ & $-0.0028(8)$ & $-0.0053(9)$ \\
\hline O6B & $0.0225(8)$ & $0.0239(8)$ & $0.0200(8)$ & $0.0048(6)$ & $0.0084(6)$ & $0.0045(6)$ \\
\hline C6B & $0.0212(11)$ & $0.0186(11)$ & $0.0151(10)$ & $0.0027(8)$ & $0.0002(8)$ & $-0.0061(8)$ \\
\hline O7B & $0.0264(8)$ & $0.0281(9)$ & $0.0207(8)$ & $0.0003(7)$ & $0.0018(6)$ & $0.0081(7)$ \\
\hline $\mathrm{C} 7 \mathrm{~B}$ & $0.0246(11)$ & $0.0175(10)$ & $0.0181(11)$ & $-0.0018(8)$ & $0.0055(8)$ & $-0.0025(8)$ \\
\hline $\mathrm{C} 8 \mathrm{~B}$ & $0.0204(10)$ & $0.0202(11)$ & $0.0175(11)$ & $0.0020(8)$ & $0.0054(8)$ & $-0.0025(8)$ \\
\hline C9B & $0.0305(12)$ & $0.0183(11)$ & $0.0208(11)$ & $0.0022(9)$ & $0.0098(9)$ & $-0.0036(8)$ \\
\hline C10B & $0.0506(16)$ & $0.0338(14)$ & $0.0241(13)$ & $0.0162(12)$ & $0.0067(11)$ & $0.0026(11)$ \\
\hline C11B & $0.0174(10)$ & $0.0146(10)$ & $0.0151(10)$ & $0.0013(7)$ & $0.0018(8)$ & $0.0003(7)$ \\
\hline C12B & $0.0156(10)$ & $0.0195(11)$ & $0.0220(11)$ & $0.0002(8)$ & $0.0023(8)$ & $-0.0036(8)$ \\
\hline C13B & $0.0149(10)$ & $0.0209(11)$ & $0.0174(11)$ & $0.0028(8)$ & $0.0028(8)$ & $0.0004(8)$ \\
\hline C14B & $0.0160(9)$ & $0.0167(10)$ & $0.0210(11)$ & $0.0035(8)$ & $0.0040(8)$ & $-0.0011(8)$ \\
\hline C15B & $0.0201(10)$ & $0.0200(11)$ & $0.0280(12)$ & $0.0021(8)$ & $0.0034(9)$ & $0.0031(9)$ \\
\hline $\mathrm{C} 16 \mathrm{~B}$ & $0.0261(12)$ & $0.0417(15)$ & $0.0298(13)$ & $-0.0035(11)$ & $-0.0019(10)$ & $0.0187(11)$ \\
\hline C17B & $0.0263(12)$ & $0.0291(13)$ & $0.0281(12)$ & $0.0092(10)$ & $0.0118(9)$ & $0.0021(10)$ \\
\hline
\end{tabular}

Geometric parameters $(\AA, \circ)$

\begin{tabular}{llll}
\hline O1A-C4A & $1.238(3)$ & O1B-C4B & $1.237(3)$ \\
N1A-C1A & $1.371(3)$ & N1B-C1B & $1.374(3)$ \\
N1A-C4A & $1.397(3)$ & N1B-C4B & $1.393(3)$ \\
N1A-H1A & 0.8800 & N1B-H1B & 0.8800 \\
C1A-N2A & $1.321(3)$ & C1B-N2B & $1.323(3)$ \\
C1A-N5A & $1.357(3)$ & C1B-N5B & $1.356(3)$
\end{tabular}




\begin{tabular}{|c|c|c|c|}
\hline $\mathrm{O} 2 \mathrm{~A}-\mathrm{C} 6 \mathrm{~A}$ & $1.425(3)$ & $\mathrm{O} 2 \mathrm{~B}-\mathrm{C} 6 \mathrm{~B}$ & $1.409(3)$ \\
\hline $\mathrm{O} 2 \mathrm{~A}-\mathrm{C} 9 \mathrm{~A}$ & $1.450(3)$ & $\mathrm{O} 2 \mathrm{~B}-\mathrm{C} 9 \mathrm{~B}$ & $1.432(3)$ \\
\hline $\mathrm{N} 2 \mathrm{~A}-\mathrm{C} 2 \mathrm{~A}$ & $1.353(3)$ & $\mathrm{N} 2 \mathrm{~B}-\mathrm{C} 2 \mathrm{~B}$ & $1.356(3)$ \\
\hline $\mathrm{C} 2 \mathrm{~A}-\mathrm{N} 4 \mathrm{~A}$ & $1.377(3)$ & $\mathrm{C} 2 \mathrm{~B}-\mathrm{C} 3 \mathrm{~B}$ & $1.382(3)$ \\
\hline $\mathrm{C} 2 \mathrm{~A}-\mathrm{C} 3 \mathrm{~A}$ & $1.380(3)$ & $\mathrm{C} 2 \mathrm{~B}-\mathrm{N} 4 \mathrm{~B}$ & $1.382(3)$ \\
\hline $\mathrm{O} 3 \mathrm{~A}-\mathrm{C} 8 \mathrm{~A}$ & $1.427(3)$ & $\mathrm{O} 3 \mathrm{~B}-\mathrm{C} 8 \mathrm{~B}$ & $1.429(3)$ \\
\hline $\mathrm{O} 3 \mathrm{~A}-\mathrm{H} 3 \mathrm{~A}$ & 0.8400 & $\mathrm{O} 3 \mathrm{~B}-\mathrm{H} 3 \mathrm{~B}$ & 0.8400 \\
\hline $\mathrm{N} 3 \mathrm{~A}-\mathrm{C} 5 \mathrm{~A}$ & $1.309(3)$ & $\mathrm{N} 3 \mathrm{~B}-\mathrm{C} 5 \mathrm{~B}$ & $1.308(3)$ \\
\hline $\mathrm{N} 3 \mathrm{~A}-\mathrm{C} 3 \mathrm{~A}$ & $1.382(3)$ & $\mathrm{N} 3 \mathrm{~B}-\mathrm{C} 3 \mathrm{~B}$ & $1.379(3)$ \\
\hline $\mathrm{C} 3 \mathrm{~A}-\mathrm{C} 4 \mathrm{~A}$ & $1.420(3)$ & $\mathrm{C} 3 \mathrm{~B}-\mathrm{C} 4 \mathrm{~B}$ & $1.420(3)$ \\
\hline $\mathrm{O} 4 \mathrm{~A}-\mathrm{C} 10 \mathrm{~A}$ & $1.417(3)$ & $\mathrm{O} 4 \mathrm{~B}-\mathrm{C} 10 \mathrm{~B}$ & $1.405(4)$ \\
\hline $\mathrm{O} 4 \mathrm{~A}-\mathrm{H} 4 \mathrm{~A}$ & 0.8400 & $\mathrm{O} 4 \mathrm{~B}-\mathrm{H} 4 \mathrm{~B}$ & 0.8400 \\
\hline $\mathrm{N} 4 \mathrm{~A}-\mathrm{C} 5 \mathrm{~A}$ & $1.376(3)$ & $\mathrm{N} 4 \mathrm{~B}-\mathrm{C} 5 \mathrm{~B}$ & $1.373(3)$ \\
\hline $\mathrm{N} 4 \mathrm{~A}-\mathrm{C} 6 \mathrm{~A}$ & $1.460(3)$ & $\mathrm{N} 4 \mathrm{~B}-\mathrm{C} 6 \mathrm{~B}$ & $1.452(3)$ \\
\hline $\mathrm{O} 5 \mathrm{~A}-\mathrm{C} 14 \mathrm{~A}$ & $1.443(2)$ & $\mathrm{O} 5 \mathrm{~B}-\mathrm{C} 11 \mathrm{~B}$ & $1.446(3)$ \\
\hline $\mathrm{O} 5 \mathrm{~A}-\mathrm{C} 11 \mathrm{~A}$ & $1.452(3)$ & $\mathrm{O} 5 \mathrm{~B}-\mathrm{C} 14 \mathrm{~B}$ & $1.447(2)$ \\
\hline $\mathrm{N} 5 \mathrm{~A}-\mathrm{C} 11 \mathrm{~A}$ & $1.433(3)$ & $\mathrm{N} 5 \mathrm{~B}-\mathrm{C} 11 \mathrm{~B}$ & $1.433(3)$ \\
\hline $\mathrm{N} 5 \mathrm{~A}-\mathrm{H} 5 \mathrm{~A}$ & 0.8800 & $\mathrm{~N} 5 \mathrm{~B}-\mathrm{H} 5 \mathrm{~B}$ & 0.8800 \\
\hline $\mathrm{C} 5 \mathrm{~A}-\mathrm{H} 5 \mathrm{~A} 1$ & 0.9500 & $\mathrm{C} 5 \mathrm{~B}-\mathrm{H} 5 \mathrm{~B} 1$ & 0.9500 \\
\hline $\mathrm{O} 6 \mathrm{~A}-\mathrm{C} 13 \mathrm{~A}$ & $1.420(3)$ & $\mathrm{O} 6 \mathrm{~B}-\mathrm{C} 13 \mathrm{~B}$ & $1.420(3)$ \\
\hline $\mathrm{O} 6 \mathrm{~A}-\mathrm{C} 17 \mathrm{~A}$ & $1.421(3)$ & $\mathrm{O} 6 \mathrm{~B}-\mathrm{C} 17 \mathrm{~B}$ & $1.426(3)$ \\
\hline $\mathrm{C} 6 \mathrm{~A}-\mathrm{C} 7 \mathrm{~A}$ & $1.515(3)$ & $\mathrm{C} 6 \mathrm{~B}-\mathrm{C} 7 \mathrm{~B}$ & $1.513(3)$ \\
\hline C6A-H6A & 1.0000 & $\mathrm{C} 6 \mathrm{~B}-\mathrm{H} 6 \mathrm{~B}$ & 1.0000 \\
\hline $\mathrm{O} 7 \mathrm{~A}-\mathrm{C} 15 \mathrm{~A}$ & $1.412(3)$ & $\mathrm{O} 7 \mathrm{~B}-\mathrm{C} 15 \mathrm{~B}$ & $1.413(3)$ \\
\hline $\mathrm{O} 7 \mathrm{~A}-\mathrm{C} 16 \mathrm{~A}$ & $1.431(3)$ & $\mathrm{O} 7 \mathrm{~B}-\mathrm{C} 16 \mathrm{~B}$ & $1.424(3)$ \\
\hline $\mathrm{C} 7 \mathrm{~A}-\mathrm{C} 8 \mathrm{~A}$ & $1.520(3)$ & $\mathrm{C} 7 \mathrm{~B}-\mathrm{C} 8 \mathrm{~B}$ & $1.525(3)$ \\
\hline $\mathrm{C} 7 \mathrm{~A}-\mathrm{H} 7 \mathrm{~A} 1$ & 0.9900 & $\mathrm{C} 7 \mathrm{~B}-\mathrm{H} 7 \mathrm{~B} 1$ & 0.9900 \\
\hline $\mathrm{C} 7 \mathrm{~A}-\mathrm{H} 7 \mathrm{~A} 2$ & 0.9900 & $\mathrm{C} 7 \mathrm{~B}-\mathrm{H} 7 \mathrm{~B} 2$ & 0.9900 \\
\hline $\mathrm{C} 8 \mathrm{~A}-\mathrm{C} 9 \mathrm{~A}$ & $1.524(3)$ & $\mathrm{C} 8 \mathrm{~B}-\mathrm{C} 9 \mathrm{~B}$ & $1.544(3)$ \\
\hline $\mathrm{C} 8 \mathrm{~A}-\mathrm{H} 8 \mathrm{~A}$ & 1.0000 & $\mathrm{C} 8 \mathrm{~B}-\mathrm{H} 8 \mathrm{~B}$ & 1.0000 \\
\hline $\mathrm{C} 9 \mathrm{~A}-\mathrm{C} 10 \mathrm{~A}$ & $1.525(3)$ & $\mathrm{C} 9 \mathrm{~B}-\mathrm{C} 10 \mathrm{~B}$ & $1.503(4)$ \\
\hline C9A-H9A & 1.0000 & $\mathrm{C} 9 \mathrm{~B}-\mathrm{H} 9 \mathrm{~B}$ & 1.0000 \\
\hline $\mathrm{C} 10 \mathrm{~A}-\mathrm{H} 10 \mathrm{~A}$ & 0.9900 & $\mathrm{C} 10 \mathrm{~B}-\mathrm{H} 10 \mathrm{C}$ & 0.9900 \\
\hline $\mathrm{C} 10 \mathrm{~A}-\mathrm{H} 10 \mathrm{~B}$ & 0.9900 & $\mathrm{C} 10 \mathrm{~B}-\mathrm{H} 10 \mathrm{D}$ & 0.9900 \\
\hline $\mathrm{C} 11 \mathrm{~A}-\mathrm{C} 12 \mathrm{~A}$ & $1.516(3)$ & $\mathrm{C} 11 \mathrm{~B}-\mathrm{C} 12 \mathrm{~B}$ & $1.514(3)$ \\
\hline $\mathrm{C} 11 \mathrm{~A}-\mathrm{H} 11 \mathrm{~A}$ & 1.0000 & $\mathrm{C} 11 \mathrm{~B}-\mathrm{H} 11 \mathrm{~B}$ & 1.0000 \\
\hline $\mathrm{C} 12 \mathrm{~A}-\mathrm{C} 13 \mathrm{~A}$ & $1.516(3)$ & $\mathrm{C} 12 \mathrm{~B}-\mathrm{C} 13 \mathrm{~B}$ & $1.525(3)$ \\
\hline $\mathrm{C} 12 \mathrm{~A}-\mathrm{H} 12 \mathrm{~A}$ & 0.9900 & $\mathrm{C} 12 \mathrm{~B}-\mathrm{H} 12 \mathrm{C}$ & 0.9900 \\
\hline $\mathrm{C} 12 \mathrm{~A}-\mathrm{H} 12 \mathrm{~B}$ & 0.9900 & $\mathrm{C} 12 \mathrm{~B}-\mathrm{H} 12 \mathrm{D}$ & 0.9900 \\
\hline $\mathrm{C} 13 \mathrm{~A}-\mathrm{C} 14 \mathrm{~A}$ & $1.544(3)$ & $\mathrm{C} 13 \mathrm{~B}-\mathrm{C} 14 \mathrm{~B}$ & $1.550(3)$ \\
\hline $\mathrm{C} 13 \mathrm{~A}-\mathrm{H} 13 \mathrm{~A}$ & 1.0000 & $\mathrm{C} 13 \mathrm{~B}-\mathrm{H} 13 \mathrm{~B}$ & 1.0000 \\
\hline $\mathrm{C} 14 \mathrm{~A}-\mathrm{C} 15 \mathrm{~A}$ & $1.512(3)$ & $\mathrm{C} 14 \mathrm{~B}-\mathrm{C} 15 \mathrm{~B}$ & $1.505(3)$ \\
\hline $\mathrm{C} 14 \mathrm{~A}-\mathrm{H} 14 \mathrm{~A}$ & 1.0000 & $\mathrm{C} 14 \mathrm{~B}-\mathrm{H} 14 \mathrm{~B}$ & 1.0000 \\
\hline $\mathrm{C} 15 \mathrm{~A}-\mathrm{H} 15 \mathrm{~A}$ & 0.9900 & $\mathrm{C} 15 \mathrm{~B}-\mathrm{H} 15 \mathrm{C}$ & 0.9900 \\
\hline $\mathrm{C} 15 \mathrm{~A}-\mathrm{H} 15 \mathrm{~B}$ & 0.9900 & $\mathrm{C} 15 \mathrm{~B}-\mathrm{H} 15 \mathrm{D}$ & 0.9900 \\
\hline $\mathrm{C} 16 \mathrm{~A}-\mathrm{H} 16 \mathrm{~A}$ & 0.9800 & $\mathrm{C} 16 \mathrm{~B}-\mathrm{H} 16 \mathrm{D}$ & 0.9800 \\
\hline $\mathrm{C} 16 \mathrm{~A}-\mathrm{H} 16 \mathrm{~B}$ & 0.9800 & $\mathrm{C} 16 \mathrm{~B}-\mathrm{H} 16 \mathrm{E}$ & 0.9800 \\
\hline $\mathrm{C} 16 \mathrm{~A}-\mathrm{H} 16 \mathrm{C}$ & 0.9800 & $\mathrm{C} 16 \mathrm{~B}-\mathrm{H} 16 \mathrm{~F}$ & 0.9800 \\
\hline
\end{tabular}




\begin{tabular}{|c|c|c|c|}
\hline $\mathrm{C} 17 \mathrm{~A}-\mathrm{H} 17 \mathrm{~A}$ & 0.9800 & $\mathrm{C} 17 \mathrm{~B}-\mathrm{H} 17 \mathrm{D}$ & 0.9800 \\
\hline $\mathrm{C} 17 \mathrm{~A}-\mathrm{H} 17 \mathrm{~B}$ & 0.9800 & $\mathrm{C} 17 \mathrm{~B}-\mathrm{H} 17 \mathrm{E}$ & 0.9800 \\
\hline $\mathrm{C} 17 \mathrm{~A}-\mathrm{H} 17 \mathrm{C}$ & 0.9800 & $\mathrm{C} 17 \mathrm{~B}-\mathrm{H} 17 \mathrm{~F}$ & 0.9800 \\
\hline $\mathrm{C} 1 \mathrm{~A}-\mathrm{N} 1 \mathrm{~A}-\mathrm{C} 4 \mathrm{~A}$ & $124.64(17)$ & $\mathrm{C} 1 \mathrm{~B}-\mathrm{N} 1 \mathrm{~B}-\mathrm{C} 4 \mathrm{~B}$ & $124.95(17)$ \\
\hline $\mathrm{C} 1 \mathrm{~A}-\mathrm{N} 1 \mathrm{~A}-\mathrm{H} 1 \mathrm{~A}$ & 117.7 & $\mathrm{C} 1 \mathrm{~B}-\mathrm{N} 1 \mathrm{~B}-\mathrm{H} 1 \mathrm{~B}$ & 117.5 \\
\hline $\mathrm{C} 4 \mathrm{~A}-\mathrm{N} 1 \mathrm{~A}-\mathrm{H} 1 \mathrm{~A}$ & 117.7 & $\mathrm{C} 4 \mathrm{~B}-\mathrm{N} 1 \mathrm{~B}-\mathrm{H} 1 \mathrm{~B}$ & 117.5 \\
\hline $\mathrm{N} 2 \mathrm{~A}-\mathrm{C} 1 \mathrm{~A}-\mathrm{N} 5 \mathrm{~A}$ & $119.73(18)$ & $\mathrm{N} 2 \mathrm{~B}-\mathrm{C} 1 \mathrm{~B}-\mathrm{N} 5 \mathrm{~B}$ & $120.96(18)$ \\
\hline $\mathrm{N} 2 \mathrm{~A}-\mathrm{C} 1 \mathrm{~A}-\mathrm{N} 1 \mathrm{~A}$ & $124.14(18)$ & $\mathrm{N} 2 \mathrm{~B}-\mathrm{C} 1 \mathrm{~B}-\mathrm{N} 1 \mathrm{~B}$ & 123.93 \\
\hline $\mathrm{N} 5 \mathrm{~A}-\mathrm{C} 1 \mathrm{~A}-\mathrm{N} 1 \mathrm{~A}$ & $116.13(17)$ & $\mathrm{N} 5 \mathrm{~B}-\mathrm{C} 1 \mathrm{~B}-\mathrm{N} 1 \mathrm{~B}$ & $115.11(18)$ \\
\hline $\mathrm{C} 6 \mathrm{~A}-\mathrm{O} 2 \mathrm{~A}-\mathrm{C} 9 \mathrm{~A}$ & $109.66(16)$ & $\mathrm{C} 6 \mathrm{~B}-\mathrm{O} 2 \mathrm{~B}-\mathrm{C} 9 \mathrm{~B}$ & $109.15(17)$ \\
\hline $\mathrm{C} 1 \mathrm{~A}-\mathrm{N} 2 \mathrm{~A}-\mathrm{C} 2 \mathrm{~A}$ & $112.51(17)$ & $\mathrm{C} 1 \mathrm{~B}-\mathrm{N} 2 \mathrm{~B}-\mathrm{C} 2 \mathrm{~B}$ & $112.52(17)$ \\
\hline $\mathrm{N} 2 \mathrm{~A}-\mathrm{C} 2 \mathrm{~A}-\mathrm{N} 4 \mathrm{~A}$ & $126.92(19)$ & $\mathrm{N} 2 \mathrm{~B}-\mathrm{C} 2 \mathrm{~B}-\mathrm{C} 3 \mathrm{~B}$ & $127.64(19)$ \\
\hline $\mathrm{N} 2 \mathrm{~A}-\mathrm{C} 2 \mathrm{~A}-\mathrm{C} 3 \mathrm{~A}$ & $127.70(19)$ & $\mathrm{N} 2 \mathrm{~B}-\mathrm{C} 2 \mathrm{~B}-\mathrm{N} 4 \mathrm{~B}$ & $127.58(19)$ \\
\hline $\mathrm{N} 4 \mathrm{~A}-\mathrm{C} 2 \mathrm{~A}-\mathrm{C} 3 \mathrm{~A}$ & $105.38(18)$ & $\mathrm{C} 3 \mathrm{~B}-\mathrm{C} 2 \mathrm{~B}-\mathrm{N} 4 \mathrm{~B}$ & $104.73(18)$ \\
\hline $\mathrm{C} 8 \mathrm{~A}-\mathrm{O} 3 \mathrm{~A}-\mathrm{H} 3 \mathrm{~A}$ & 109.5 & $\mathrm{C} 8 \mathrm{~B}-\mathrm{O} 3 \mathrm{~B}-\mathrm{H} 3 \mathrm{~B}$ & 109.5 \\
\hline $\mathrm{C} 5 \mathrm{~A}-\mathrm{N} 3 \mathrm{~A}-\mathrm{C} 3 \mathrm{~A}$ & $104.51(18)$ & $\mathrm{C} 5 \mathrm{~B}-\mathrm{N} 3 \mathrm{~B}-\mathrm{C} 3 \mathrm{~B}$ & $104.43(17)$ \\
\hline $\mathrm{C} 2 \mathrm{~A}-\mathrm{C} 3 \mathrm{~A}-\mathrm{N} 3 \mathrm{~A}$ & $110.89(18)$ & $\mathrm{N} 3 \mathrm{~B}-\mathrm{C} 3 \mathrm{~B}-\mathrm{C} 2 \mathrm{~B}$ & $111.34(19)$ \\
\hline $\mathrm{C} 2 \mathrm{~A}-\mathrm{C} 3 \mathrm{~A}-\mathrm{C} 4 \mathrm{~A}$ & $119.30(18)$ & $\mathrm{N} 3 \mathrm{~B}-\mathrm{C} 3 \mathrm{~B}-\mathrm{C} 4 \mathrm{~B}$ & $129.16(19)$ \\
\hline $\mathrm{N} 3 \mathrm{~A}-\mathrm{C} 3 \mathrm{~A}-\mathrm{C} 4 \mathrm{~A}$ & $129.73(19)$ & $\mathrm{C} 2 \mathrm{~B}-\mathrm{C} 3 \mathrm{~B}-\mathrm{C} 4 \mathrm{~B}$ & $119.33(19)$ \\
\hline $\mathrm{C} 10 \mathrm{~A}-\mathrm{O} 4 \mathrm{~A}-\mathrm{H} 4 \mathrm{~A}$ & 109.5 & $\mathrm{C} 10 \mathrm{~B}-\mathrm{O} 4 \mathrm{~B}-\mathrm{H} 4 \mathrm{~B}$ & 109.5 \\
\hline $\mathrm{C} 5 \mathrm{~A}-\mathrm{N} 4 \mathrm{~A}-\mathrm{C} 2 \mathrm{~A}$ & $106.28(17)$ & $\mathrm{C} 5 \mathrm{~B}-\mathrm{N} 4 \mathrm{~B}-\mathrm{C} 2 \mathrm{~B}$ & $106.50(17)$ \\
\hline $\mathrm{C} 5 \mathrm{~A}-\mathrm{N} 4 \mathrm{~A}-\mathrm{C} 6 \mathrm{~A}$ & $124.53(18)$ & $\mathrm{C} 5 \mathrm{~B}-\mathrm{N} 4 \mathrm{~B}-\mathrm{C} 6 \mathrm{~B}$ & $123.40(18)$ \\
\hline $\mathrm{C} 2 \mathrm{~A}-\mathrm{N} 4 \mathrm{~A}-\mathrm{C} 6 \mathrm{~A}$ & $128.96(18)$ & $\mathrm{C} 2 \mathrm{~B}-\mathrm{N} 4 \mathrm{~B}-\mathrm{C} 6 \mathrm{~B}$ & $129.95(18)$ \\
\hline $\mathrm{O} 1 \mathrm{~A}-\mathrm{C} 4 \mathrm{~A}-\mathrm{N} 1 \mathrm{~A}$ & $121.03(18)$ & $\mathrm{O} 1 \mathrm{~B}-\mathrm{C} 4 \mathrm{~B}-\mathrm{N} 1 \mathrm{~B}$ & $121.23(19)$ \\
\hline $\mathrm{O} 1 \mathrm{~A}-\mathrm{C} 4 \mathrm{~A}-\mathrm{C} 3 \mathrm{~A}$ & $127.38(19)$ & $\mathrm{O} 1 \mathrm{~B}-\mathrm{C} 4 \mathrm{~B}-\mathrm{C} 3 \mathrm{~B}$ & $127.16(19)$ \\
\hline $\mathrm{N} 1 \mathrm{~A}-\mathrm{C} 4 \mathrm{~A}-\mathrm{C} 3 \mathrm{~A}$ & $111.59(17)$ & $\mathrm{N} 1 \mathrm{~B}-\mathrm{C} 4 \mathrm{~B}-\mathrm{C} 3 \mathrm{~B}$ & $111.59(17)$ \\
\hline $\mathrm{C} 14 \mathrm{~A}-\mathrm{O} 5 \mathrm{~A}-\mathrm{C} 11 \mathrm{~A}$ & $109.25(15)$ & $\mathrm{C} 11 \mathrm{~B}-\mathrm{O} 5 \mathrm{~B}-\mathrm{C} 14 \mathrm{~B}$ & $106.31(15)$ \\
\hline $\mathrm{C} 1 \mathrm{~A}-\mathrm{N} 5 \mathrm{~A}-\mathrm{C} 11 \mathrm{~A}$ & $121.20(17)$ & $\mathrm{C} 1 \mathrm{~B}-\mathrm{N} 5 \mathrm{~B}-\mathrm{C} 11 \mathrm{~B}$ & $121.92(18)$ \\
\hline $\mathrm{C} 1 \mathrm{~A}-\mathrm{N} 5 \mathrm{~A}-\mathrm{H} 5 \mathrm{~A}$ & 119.4 & $\mathrm{C} 1 \mathrm{~B}-\mathrm{N} 5 \mathrm{~B}-\mathrm{H} 5 \mathrm{~B}$ & 119.0 \\
\hline $\mathrm{C} 11 \mathrm{~A}-\mathrm{N} 5 \mathrm{~A}-\mathrm{H} 5 \mathrm{~A}$ & 119.4 & $\mathrm{C} 11 \mathrm{~B}-\mathrm{N} 5 \mathrm{~B}-\mathrm{H} 5 \mathrm{~B}$ & 119.0 \\
\hline $\mathrm{N} 3 \mathrm{~A}-\mathrm{C} 5 \mathrm{~A}-\mathrm{N} 4 \mathrm{~A}$ & $112.93(18)$ & $\mathrm{N} 3 \mathrm{~B}-\mathrm{C} 5 \mathrm{~B}-\mathrm{N} 4 \mathrm{~B}$ & $112.99(18)$ \\
\hline $\mathrm{N} 3 \mathrm{~A}-\mathrm{C} 5 \mathrm{~A}-\mathrm{H} 5 \mathrm{~A} 1$ & 123.5 & $\mathrm{~N} 3 \mathrm{~B}-\mathrm{C} 5 \mathrm{~B}-\mathrm{H} 5 \mathrm{~B} 1$ & 123.5 \\
\hline $\mathrm{N} 4 \mathrm{~A}-\mathrm{C} 5 \mathrm{~A}-\mathrm{H} 5 \mathrm{~A} 1$ & 123.5 & $\mathrm{~N} 4 \mathrm{~B}-\mathrm{C} 5 \mathrm{~B}-\mathrm{H} 5 \mathrm{~B} 1$ & 123.5 \\
\hline $\mathrm{C} 13 \mathrm{~A}-\mathrm{O} 6 \mathrm{~A}-\mathrm{C} 17 \mathrm{~A}$ & $111.90(16)$ & $\mathrm{C} 13 \mathrm{~B}-\mathrm{O} 6 \mathrm{~B}-\mathrm{C} 17 \mathrm{~B}$ & $112.00(17)$ \\
\hline $\mathrm{O} 2 \mathrm{~A}-\mathrm{C} 6 \mathrm{~A}-\mathrm{N} 4 \mathrm{~A}$ & $108.59(17)$ & $\mathrm{O} 2 \mathrm{~B}-\mathrm{C} 6 \mathrm{~B}-\mathrm{N} 4 \mathrm{~B}$ & $107.89(18)$ \\
\hline $\mathrm{O} 2 \mathrm{~A}-\mathrm{C} 6 \mathrm{~A}-\mathrm{C} 7 \mathrm{~A}$ & $106.39(17)$ & $\mathrm{O} 2 \mathrm{~B}-\mathrm{C} 6 \mathrm{~B}-\mathrm{C} 7 \mathrm{~B}$ & $105.90(17)$ \\
\hline $\mathrm{N} 4 \mathrm{~A}-\mathrm{C} 6 \mathrm{~A}-\mathrm{C} 7 \mathrm{~A}$ & $115.46(18)$ & $\mathrm{N} 4 \mathrm{~B}-\mathrm{C} 6 \mathrm{~B}-\mathrm{C} 7 \mathrm{~B}$ & $116.03(18)$ \\
\hline $\mathrm{O} 2 \mathrm{~A}-\mathrm{C} 6 \mathrm{~A}-\mathrm{H} 6 \mathrm{~A}$ & 108.7 & $\mathrm{O} 2 \mathrm{~B}-\mathrm{C} 6 \mathrm{~B}-\mathrm{H} 6 \mathrm{~B}$ & 108.9 \\
\hline $\mathrm{N} 4 \mathrm{~A}-\mathrm{C} 6 \mathrm{~A}-\mathrm{H} 6 \mathrm{~A}$ & 108.7 & $\mathrm{~N} 4 \mathrm{~B}-\mathrm{C} 6 \mathrm{~B}-\mathrm{H} 6 \mathrm{~B}$ & 108.9 \\
\hline $\mathrm{C} 7 \mathrm{~A}-\mathrm{C} 6 \mathrm{~A}-\mathrm{H} 6 \mathrm{~A}$ & 108.7 & $\mathrm{C} 7 \mathrm{~B}-\mathrm{C} 6 \mathrm{~B}-\mathrm{H} 6 \mathrm{~B}$ & 108.9 \\
\hline $\mathrm{C} 15 \mathrm{~A}-\mathrm{O} 7 \mathrm{~A}-\mathrm{C} 16 \mathrm{~A}$ & $112.43(18)$ & $\mathrm{C} 15 \mathrm{~B}-\mathrm{O} 7 \mathrm{~B}-\mathrm{C} 16 \mathrm{~B}$ & $110.76(19)$ \\
\hline $\mathrm{C} 6 \mathrm{~A}-\mathrm{C} 7 \mathrm{~A}-\mathrm{C} 8 \mathrm{~A}$ & $102.12(17)$ & $\mathrm{C} 6 \mathrm{~B}-\mathrm{C} 7 \mathrm{~B}-\mathrm{C} 8 \mathrm{~B}$ & $101.90(17)$ \\
\hline $\mathrm{C} 6 \mathrm{~A}-\mathrm{C} 7 \mathrm{~A}-\mathrm{H} 7 \mathrm{~A} 1$ & 111.3 & $\mathrm{C} 6 \mathrm{~B}-\mathrm{C} 7 \mathrm{~B}-\mathrm{H} 7 \mathrm{~B} 1$ & 111.4 \\
\hline $\mathrm{C} 8 \mathrm{~A}-\mathrm{C} 7 \mathrm{~A}-\mathrm{H} 7 \mathrm{~A} 1$ & 111.3 & $\mathrm{C} 8 \mathrm{~B}-\mathrm{C} 7 \mathrm{~B}-\mathrm{H} 7 \mathrm{~B} 1$ & 111.4 \\
\hline $\mathrm{C} 6 \mathrm{~A}-\mathrm{C} 7 \mathrm{~A}-\mathrm{H} 7 \mathrm{~A} 2$ & 111.3 & $\mathrm{C} 6 \mathrm{~B}-\mathrm{C} 7 \mathrm{~B}-\mathrm{H} 7 \mathrm{~B} 2$ & 111.4 \\
\hline $\mathrm{C} 8 \mathrm{~A}-\mathrm{C} 7 \mathrm{~A}-\mathrm{H} 7 \mathrm{~A} 2$ & 111.3 & $\mathrm{C} 8 \mathrm{~B}-\mathrm{C} 7 \mathrm{~B}-\mathrm{H} 7 \mathrm{~B} 2$ & 111.4 \\
\hline $\mathrm{H} 7 \mathrm{~A} 1-\mathrm{C} 7 \mathrm{~A}-\mathrm{H} 7 \mathrm{~A} 2$ & 109.2 & $\mathrm{H} 7 \mathrm{~B} 1-\mathrm{C} 7 \mathrm{~B}-\mathrm{H} 7 \mathrm{~B} 2$ & 109.3 \\
\hline
\end{tabular}




$\begin{array}{ll}\text { O3A-C8A-C7A } & 111.70(18) \\ \text { O3A-C8A-C9A } & 109.09(17) \\ \text { C7A-C8A-C9A } & 102.03(17) \\ \text { O3A-C8A-H8A } & 111.2 \\ \text { C7A-C8A-H8A } & 111.2 \\ \text { C9A-C8A-H8A } & 111.2 \\ \text { O2A-C9A-C8A } & 105.75(17) \\ \text { O2A-C9A-C10A } & 108.82(17) \\ \text { C8A-C9A-C10A } & 114.59(18) \\ \text { O2A-C9A-H9A } & 109.2 \\ \text { C8A-C9A-H9A } & 109.2 \\ \text { C10A-C9A-H9A } & 109.2 \\ \text { O4A-C10A-C9A } & 111.51(19) \\ \text { O4A-C10A-H10A } & 109.3 \\ \text { C9A-C10A-H10A } & 109.3 \\ \text { O4A-C10A-H10B } & 109.3 \\ \text { C9A-C10A-H10B } & 109.3 \\ \text { H10A-C10A-H10B } & 108.0 \\ \text { N5A-C11A-O5A } & 109.19(17) \\ \text { N5A-C11A-C12A } & 113.26(17) \\ \text { O5A-C11A-C12A } & 104.50(16) \\ \text { N5A-C11A-H11A } & 109.9 \\ \text { O5A-C11A-H11A } & 109.9 \\ \text { C12A-C11A-H11A } & 109.9 \\ \text { C13A-C12A-C11A } & 103.50(17) \\ \text { C13A-C12A-H12A } & 111.1 \\ \text { C11A-C12A-H12A } & 111.1 \\ \text { C13A-C12A-H12B } & 111.1 \\ \text { C11A-C12A-H12B } & 111.1 \\ \text { H12A-C12A-H12B } & 109.0 \\ \text { O6A-C13A-C12A } & 109.43(17) \\ \text { O6A-C13A-C14A } & 112.71(17) \\ \text { C12A-C13A-C14A } & 103.35(16) \\ \text { O6A-C13A-H13A } & 110.4 \\ \text { C12A-C13A-H13A } & 110.4 \\ \text { C14A-C13A-H13A } & 110.4 \\ \text { O5A-C14A-C15A } & 109.66(17) \\ \text { O5A-C14A-C13A } & 106.95(16) \\ \text { C15A-C14A-C13A } & 112.09(17) \\ \text { O5A-C14A-H14A } & 109.4 \\ \text { C15A-C14A-H14A } & 109.4 \\ \text { C13A-C14A-H14A } & 109.4 \\ \text { O7A-C15A-C14A } & 107.74(17) \\ \text { O7A-C15A-H15A } & 110.2 \\ \text { C14A-C15A-H15A } & 110.2 \\ \text { O7A-C15A-H15B } & 110.2 \\ \text { C14A-C15A-H15B } & 110.2 \\ \text { H15A-C15A-H15B } & 108.5 \\ & \end{array}$

\begin{tabular}{|c|c|}
\hline $\mathrm{O} 3 \mathrm{~B}-\mathrm{C} 8 \mathrm{~B}-\mathrm{C} 7 \mathrm{~B}$ & $111.64(18)$ \\
\hline $\mathrm{O} 3 \mathrm{~B}-\mathrm{C} 8 \mathrm{~B}-\mathrm{C} 9 \mathrm{~B}$ & $107.79(17)$ \\
\hline $\mathrm{C} 7 \mathrm{~B}-\mathrm{C} 8 \mathrm{~B}-\mathrm{C} 9 \mathrm{~B}$ & $102.92(17)$ \\
\hline $\mathrm{O} 3 \mathrm{~B}-\mathrm{C} 8 \mathrm{~B}-\mathrm{H} 8 \mathrm{~B}$ & 111.4 \\
\hline $\mathrm{C} 7 \mathrm{~B}-\mathrm{C} 8 \mathrm{~B}-\mathrm{H} 8 \mathrm{~B}$ & 111.4 \\
\hline $\mathrm{C} 9 \mathrm{~B}-\mathrm{C} 8 \mathrm{~B}-\mathrm{H} 8 \mathrm{~B}$ & 111.4 \\
\hline $\mathrm{O} 2 \mathrm{~B}-\mathrm{C} 9 \mathrm{~B}-\mathrm{C} 10 \mathrm{~B}$ & $107.12(19)$ \\
\hline $\mathrm{O} 2 \mathrm{~B}-\mathrm{C} 9 \mathrm{~B}-\mathrm{C} 8 \mathrm{~B}$ & $107.00(17)$ \\
\hline $\mathrm{C} 10 \mathrm{~B}-\mathrm{C} 9 \mathrm{~B}-\mathrm{C} 8 \mathrm{~B}$ & $114.3(2)$ \\
\hline $\mathrm{O} 2 \mathrm{~B}-\mathrm{C} 9 \mathrm{~B}-\mathrm{H} 9 \mathrm{~B}$ & 109.4 \\
\hline $\mathrm{C} 10 \mathrm{~B}-\mathrm{C} 9 \mathrm{~B}-\mathrm{H} 9 \mathrm{~B}$ & 109.4 \\
\hline $\mathrm{C} 8 \mathrm{~B}-\mathrm{C} 9 \mathrm{~B}-\mathrm{H} 9 \mathrm{~B}$ & 109.4 \\
\hline $\mathrm{O} 4 \mathrm{~B}-\mathrm{C} 10 \mathrm{~B}-\mathrm{C} 9 \mathrm{~B}$ & $111.9(2)$ \\
\hline $\mathrm{O} 4 \mathrm{~B}-\mathrm{C} 10 \mathrm{~B}-\mathrm{H} 10 \mathrm{C}$ & 109.2 \\
\hline $\mathrm{C} 9 \mathrm{~B}-\mathrm{C} 10 \mathrm{~B}-\mathrm{H} 10 \mathrm{C}$ & 109.2 \\
\hline $\mathrm{O} 4 \mathrm{~B}-\mathrm{C} 10 \mathrm{~B}-\mathrm{H} 10 \mathrm{D}$ & 109.2 \\
\hline $\mathrm{C} 9 \mathrm{~B}-\mathrm{C} 10 \mathrm{~B}-\mathrm{H} 10 \mathrm{D}$ & 109.2 \\
\hline $\mathrm{H} 10 \mathrm{C}-\mathrm{C} 10 \mathrm{~B}-\mathrm{H} 10 \mathrm{D}$ & 107.9 \\
\hline $\mathrm{N} 5 \mathrm{~B}-\mathrm{C} 11 \mathrm{~B}-\mathrm{O} 5 \mathrm{~B}$ & $109.36(17)$ \\
\hline $\mathrm{N} 5 \mathrm{~B}-\mathrm{C} 11 \mathrm{~B}-\mathrm{C} 12 \mathrm{~B}$ & $115.06(18)$ \\
\hline $\mathrm{O} 5 \mathrm{~B}-\mathrm{C} 11 \mathrm{~B}-\mathrm{C} 12 \mathrm{~B}$ & $102.84(16)$ \\
\hline $\mathrm{N} 5 \mathrm{~B}-\mathrm{C} 11 \mathrm{~B}-\mathrm{H} 11 \mathrm{~B}$ & 109.8 \\
\hline $\mathrm{O} 5 \mathrm{~B}-\mathrm{C} 11 \mathrm{~B}-\mathrm{H} 11 \mathrm{~B}$ & 109.8 \\
\hline $\mathrm{C} 12 \mathrm{~B}-\mathrm{C} 11 \mathrm{~B}-\mathrm{H} 11 \mathrm{~B}$ & 109.8 \\
\hline $\mathrm{C} 11 \mathrm{~B}-\mathrm{C} 12 \mathrm{~B}-\mathrm{C} 13 \mathrm{~B}$ & $101.47(17)$ \\
\hline $\mathrm{C} 11 \mathrm{~B}-\mathrm{C} 12 \mathrm{~B}-\mathrm{H} 12 \mathrm{C}$ & 111.5 \\
\hline $\mathrm{C} 13 \mathrm{~B}-\mathrm{C} 12 \mathrm{~B}-\mathrm{H} 12 \mathrm{C}$ & 111.5 \\
\hline $\mathrm{C} 11 \mathrm{~B}-\mathrm{C} 12 \mathrm{~B}-\mathrm{H} 12 \mathrm{D}$ & 111.5 \\
\hline $\mathrm{C} 13 \mathrm{~B}-\mathrm{C} 12 \mathrm{~B}-\mathrm{H} 12 \mathrm{D}$ & 111.5 \\
\hline $\mathrm{H} 12 \mathrm{C}-\mathrm{C} 12 \mathrm{~B}-\mathrm{H} 12 \mathrm{D}$ & 109.3 \\
\hline $\mathrm{O} 6 \mathrm{~B}-\mathrm{C} 13 \mathrm{~B}-\mathrm{C} 12 \mathrm{~B}$ & $108.29(17)$ \\
\hline $\mathrm{O} 6 \mathrm{~B}-\mathrm{C} 13 \mathrm{~B}-\mathrm{C} 14 \mathrm{~B}$ & $111.87(18)$ \\
\hline $\mathrm{C} 12 \mathrm{~B}-\mathrm{C} 13 \mathrm{~B}-\mathrm{C} 14 \mathrm{~B}$ & $103.26(16)$ \\
\hline $\mathrm{O} 6 \mathrm{~B}-\mathrm{C} 13 \mathrm{~B}-\mathrm{H} 13 \mathrm{~B}$ & 111.0 \\
\hline $\mathrm{C} 12 \mathrm{~B}-\mathrm{C} 13 \mathrm{~B}-\mathrm{H} 13 \mathrm{~B}$ & 111.0 \\
\hline $\mathrm{C} 14 \mathrm{~B}-\mathrm{C} 13 \mathrm{~B}-\mathrm{H} 13 \mathrm{~B}$ & 111.0 \\
\hline $\mathrm{O} 5 \mathrm{~B}-\mathrm{C} 14 \mathrm{~B}-\mathrm{C} 15 \mathrm{~B}$ & $110.05(17)$ \\
\hline $\mathrm{O} 5 \mathrm{~B}-\mathrm{C} 14 \mathrm{~B}-\mathrm{C} 13 \mathrm{~B}$ & $106.37(16)$ \\
\hline $\mathrm{C} 15 \mathrm{~B}-\mathrm{C} 14 \mathrm{~B}-\mathrm{C} 13 \mathrm{~B}$ & $114.55(18)$ \\
\hline $\mathrm{O} 5 \mathrm{~B}-\mathrm{C} 14 \mathrm{~B}-\mathrm{H} 14 \mathrm{~B}$ & 108.6 \\
\hline $\mathrm{C} 15 \mathrm{~B}-\mathrm{C} 14 \mathrm{~B}-\mathrm{H} 14 \mathrm{~B}$ & 108.6 \\
\hline $\mathrm{C} 13 \mathrm{~B}-\mathrm{C} 14 \mathrm{~B}-\mathrm{H} 14 \mathrm{~B}$ & 108.6 \\
\hline $\mathrm{O} 7 \mathrm{~B}-\mathrm{C} 15 \mathrm{~B}-\mathrm{C} 14 \mathrm{~B}$ & $109.74(18)$ \\
\hline $\mathrm{O} 7 \mathrm{~B}-\mathrm{C} 15 \mathrm{~B}-\mathrm{H} 15 \mathrm{C}$ & 109.7 \\
\hline $\mathrm{C} 14 \mathrm{~B}-\mathrm{C} 15 \mathrm{~B}-\mathrm{H} 15 \mathrm{C}$ & 109.7 \\
\hline $\mathrm{O} 7 \mathrm{~B}-\mathrm{C} 15 \mathrm{~B}-\mathrm{H} 15 \mathrm{D}$ & 109.7 \\
\hline $\mathrm{C} 14 \mathrm{~B}-\mathrm{C} 15 \mathrm{~B}-\mathrm{H} 15 \mathrm{D}$ & 109.7 \\
\hline $\mathrm{H} 15 \mathrm{C}-\mathrm{C} 15 \mathrm{~B}-\mathrm{H} 15 \mathrm{D}$ & 108.2 \\
\hline
\end{tabular}




\begin{tabular}{|c|c|}
\hline $\mathrm{O} 7 \mathrm{~A}-\mathrm{C} 16 \mathrm{~A}-\mathrm{H} 16 \mathrm{~A}$ & 109.5 \\
\hline $\mathrm{O} 7 \mathrm{~A}-\mathrm{C} 16 \mathrm{~A}-\mathrm{H} 16 \mathrm{~B}$ & 109.5 \\
\hline $\mathrm{H} 16 \mathrm{~A}-\mathrm{C} 16 \mathrm{~A}-\mathrm{H} 16 \mathrm{~B}$ & 109.5 \\
\hline $\mathrm{O} 7 \mathrm{~A}-\mathrm{C} 16 \mathrm{~A}-\mathrm{H} 16 \mathrm{C}$ & 109.5 \\
\hline $\mathrm{H} 16 \mathrm{~A}-\mathrm{C} 16 \mathrm{~A}-\mathrm{H} 16 \mathrm{C}$ & 109.5 \\
\hline $\mathrm{H} 16 \mathrm{~B}-\mathrm{C} 16 \mathrm{~A}-\mathrm{H} 16 \mathrm{C}$ & 109.5 \\
\hline $\mathrm{O} 6 \mathrm{~A}-\mathrm{C} 17 \mathrm{~A}-\mathrm{H} 17 \mathrm{~A}$ & 109.5 \\
\hline $\mathrm{O} 6 \mathrm{~A}-\mathrm{C} 17 \mathrm{~A}-\mathrm{H} 17 \mathrm{~B}$ & 109.5 \\
\hline $\mathrm{H} 17 \mathrm{~A}-\mathrm{C} 17 \mathrm{~A}-\mathrm{H} 17 \mathrm{~B}$ & 109.5 \\
\hline $\mathrm{O} 6 \mathrm{~A}-\mathrm{C} 17 \mathrm{~A}-\mathrm{H} 17 \mathrm{C}$ & 109.5 \\
\hline $\mathrm{H} 17 \mathrm{~A}-\mathrm{C} 17 \mathrm{~A}-\mathrm{H} 17 \mathrm{C}$ & 109.5 \\
\hline $\mathrm{H} 17 \mathrm{~B}-\mathrm{C} 17 \mathrm{~A}-\mathrm{H} 17 \mathrm{C}$ & 109.5 \\
\hline $\mathrm{C} 4 \mathrm{~A}-\mathrm{N} 1 \mathrm{~A}-\mathrm{C} 1 \mathrm{~A}-\mathrm{N} 2 \mathrm{~A}$ & $-1.4(3)$ \\
\hline $\mathrm{C} 4 \mathrm{~A}-\mathrm{N} 1 \mathrm{~A}-\mathrm{C} 1 \mathrm{~A}-\mathrm{N} 5 \mathrm{~A}$ & $177.84(18)$ \\
\hline $\mathrm{N} 5 \mathrm{~A}-\mathrm{C} 1 \mathrm{~A}-\mathrm{N} 2 \mathrm{~A}-\mathrm{C} 2 \mathrm{~A}$ & $-176.82(19)$ \\
\hline $\mathrm{N} 1 \mathrm{~A}-\mathrm{C} 1 \mathrm{~A}-\mathrm{N} 2 \mathrm{~A}-\mathrm{C} 2 \mathrm{~A}$ & $2.4(3)$ \\
\hline $\mathrm{C} 1 \mathrm{~A}-\mathrm{N} 2 \mathrm{~A}-\mathrm{C} 2 \mathrm{~A}-\mathrm{N} 4 \mathrm{~A}$ & $179.8(2)$ \\
\hline $\mathrm{C} 1 \mathrm{~A}-\mathrm{N} 2 \mathrm{~A}-\mathrm{C} 2 \mathrm{~A}-\mathrm{C} 3 \mathrm{~A}$ & $-0.3(3)$ \\
\hline $\mathrm{N} 2 \mathrm{~A}-\mathrm{C} 2 \mathrm{~A}-\mathrm{C} 3 \mathrm{~A}-\mathrm{N} 3 \mathrm{~A}$ & $-179.8(2)$ \\
\hline $\mathrm{N} 4 \mathrm{~A}-\mathrm{C} 2 \mathrm{~A}-\mathrm{C} 3 \mathrm{~A}-\mathrm{N} 3 \mathrm{~A}$ & $0.1(2)$ \\
\hline $\mathrm{N} 2 \mathrm{~A}-\mathrm{C} 2 \mathrm{~A}-\mathrm{C} 3 \mathrm{~A}-\mathrm{C} 4 \mathrm{~A}$ & $-2.9(3)$ \\
\hline $\mathrm{N} 4 \mathrm{~A}-\mathrm{C} 2 \mathrm{~A}-\mathrm{C} 3 \mathrm{~A}-\mathrm{C} 4 \mathrm{~A}$ & $177.03(18)$ \\
\hline $\mathrm{C} 5 \mathrm{~A}-\mathrm{N} 3 \mathrm{~A}-\mathrm{C} 3 \mathrm{~A}-\mathrm{C} 2 \mathrm{~A}$ & $0.2(2)$ \\
\hline $\mathrm{C} 5 \mathrm{~A}-\mathrm{N} 3 \mathrm{~A}-\mathrm{C} 3 \mathrm{~A}-\mathrm{C} 4 \mathrm{~A}$ & $-176.3(2)$ \\
\hline $\mathrm{N} 2 \mathrm{~A}-\mathrm{C} 2 \mathrm{~A}-\mathrm{N} 4 \mathrm{~A}-\mathrm{C} 5 \mathrm{~A}$ & $179.5(2)$ \\
\hline $\mathrm{C} 3 \mathrm{~A}-\mathrm{C} 2 \mathrm{~A}-\mathrm{N} 4 \mathrm{~A}-\mathrm{C} 5 \mathrm{~A}$ & $-0.4(2)$ \\
\hline $\mathrm{N} 2 \mathrm{~A}-\mathrm{C} 2 \mathrm{~A}-\mathrm{N} 4 \mathrm{~A}-\mathrm{C} 6 \mathrm{~A}$ & $4.8(4)$ \\
\hline $\mathrm{C} 3 \mathrm{~A}-\mathrm{C} 2 \mathrm{~A}-\mathrm{N} 4 \mathrm{~A}-\mathrm{C} 6 \mathrm{~A}$ & $-175.1(2)$ \\
\hline $\mathrm{C} 1 \mathrm{~A}-\mathrm{N} 1 \mathrm{~A}-\mathrm{C} 4 \mathrm{~A}-\mathrm{O} 1 \mathrm{~A}$ & $178.41(19)$ \\
\hline $\mathrm{C} 1 \mathrm{~A}-\mathrm{N} 1 \mathrm{~A}-\mathrm{C} 4 \mathrm{~A}-\mathrm{C} 3 \mathrm{~A}$ & $-1.7(3)$ \\
\hline $\mathrm{C} 2 \mathrm{~A}-\mathrm{C} 3 \mathrm{~A}-\mathrm{C} 4 \mathrm{~A}-\mathrm{O} 1 \mathrm{~A}$ & $-176.6(2)$ \\
\hline $\mathrm{N} 3 \mathrm{~A}-\mathrm{C} 3 \mathrm{~A}-\mathrm{C} 4 \mathrm{~A}-\mathrm{O} 1 \mathrm{~A}$ & $-0.3(4)$ \\
\hline $\mathrm{C} 2 \mathrm{~A}-\mathrm{C} 3 \mathrm{~A}-\mathrm{C} 4 \mathrm{~A}-\mathrm{N} 1 \mathrm{~A}$ & $3.6(3)$ \\
\hline $\mathrm{N} 3 \mathrm{~A}-\mathrm{C} 3 \mathrm{~A}-\mathrm{C} 4 \mathrm{~A}-\mathrm{N} 1 \mathrm{~A}$ & $179.8(2)$ \\
\hline $\mathrm{N} 2 \mathrm{~A}-\mathrm{C} 1 \mathrm{~A}-\mathrm{N} 5 \mathrm{~A}-\mathrm{C} 11 \mathrm{~A}$ & $-5.3(3)$ \\
\hline $\mathrm{N} 1 \mathrm{~A}-\mathrm{C} 1 \mathrm{~A}-\mathrm{N} 5 \mathrm{~A}-\mathrm{C} 11 \mathrm{~A}$ & $175.39(18)$ \\
\hline $\mathrm{C} 3 \mathrm{~A}-\mathrm{N} 3 \mathrm{~A}-\mathrm{C} 5 \mathrm{~A}-\mathrm{N} 4 \mathrm{~A}$ & $-0.5(3)$ \\
\hline $\mathrm{C} 2 \mathrm{~A}-\mathrm{N} 4 \mathrm{~A}-\mathrm{C} 5 \mathrm{~A}-\mathrm{N} 3 \mathrm{~A}$ & $0.6(3)$ \\
\hline $\mathrm{C} 6 \mathrm{~A}-\mathrm{N} 4 \mathrm{~A}-\mathrm{C} 5 \mathrm{~A}-\mathrm{N} 3 \mathrm{~A}$ & $175.6(2)$ \\
\hline $\mathrm{C} 9 \mathrm{~A}-\mathrm{O} 2 \mathrm{~A}-\mathrm{C} 6 \mathrm{~A}-\mathrm{N} 4 \mathrm{~A}$ & $-138.45(17)$ \\
\hline $\mathrm{C} 9 \mathrm{~A}-\mathrm{O} 2 \mathrm{~A}-\mathrm{C} 6 \mathrm{~A}-\mathrm{C} 7 \mathrm{~A}$ & $-13.6(2)$ \\
\hline $\mathrm{C} 5 \mathrm{~A}-\mathrm{N} 4 \mathrm{~A}-\mathrm{C} 6 \mathrm{~A}-\mathrm{O} 2 \mathrm{~A}$ & $-103.0(2)$ \\
\hline $\mathrm{C} 2 \mathrm{~A}-\mathrm{N} 4 \mathrm{~A}-\mathrm{C} 6 \mathrm{~A}-\mathrm{O} 2 \mathrm{~A}$ & $70.9(3)$ \\
\hline $\mathrm{C} 5 \mathrm{~A}-\mathrm{N} 4 \mathrm{~A}-\mathrm{C} 6 \mathrm{~A}-\mathrm{C} 7 \mathrm{~A}$ & $137.7(2)$ \\
\hline $\mathrm{C} 2 \mathrm{~A}-\mathrm{N} 4 \mathrm{~A}-\mathrm{C} 6 \mathrm{~A}-\mathrm{C} 7 \mathrm{~A}$ & $-48.4(3)$ \\
\hline $\mathrm{O} 2 \mathrm{~A}-\mathrm{C} 6 \mathrm{~A}-\mathrm{C} 7 \mathrm{~A}-\mathrm{C} 8 \mathrm{~A}$ & $31.8(2)$ \\
\hline $\mathrm{N} 4 \mathrm{~A}-\mathrm{C} 6 \mathrm{~A}-\mathrm{C} 7 \mathrm{~A}-\mathrm{C} 8 \mathrm{~A}$ & $152.34(18)$ \\
\hline
\end{tabular}

$\begin{array}{ll}\text { O7B-C16B-H16D } & 109.5 \\ \text { O7B-C16B-H16E } & 109.5 \\ \text { H16D-C16B-H16E } & 109.5 \\ \text { O7B-C16B-H16F } & 109.5 \\ \text { H16D-C16B-H16F } & 109.5 \\ \text { H16E-C16B-H16F } & 109.5 \\ \text { O6B-C17B-H17D } & 109.5 \\ \text { O6B-C17B-H17E } & 109.5 \\ \text { H17D-C17B-H17E } & 109.5 \\ \text { O6B-C17B-H17F } & 109.5 \\ \text { H17D-C17B-H17F } & 109.5 \\ \text { H17E-C17B-H17F } & 109.5 \\ & \\ \text { C4B-N1B-C1B-N2B } & 0.2(3) \\ \text { C4B-N1B-C1B-N5B } & -179.83(18) \\ \text { N5B-C1B-N2B-C2B } & -178.87(19) \\ \text { N1B-C1B-N2B-C2B } & 1.1(3) \\ \text { C1B-N2B-C2B-C3B } & -0.8(3) \\ \text { C1B-N2B-C2B-N4B } & -177.6(2) \\ \text { C5B-N3B-C3B-C2B } & 0.2(3) \\ \text { C5B-N3B-C3B-C4B } & -175.1(2) \\ \text { N2B-C2B-C3B-N3B } & -176.7(2) \\ \text { N4B-C2B-C3B-N3B } & 0.7(2) \\ \text { N2B-C2B-C3B-C4B } & -0.9(3) \\ \text { N4B-C2B-C3B-C4B } & 176.47(19) \\ \text { N2B-C2B-N4B-C5B } & 176.1(2) \\ \text { C3B-C2B-N4B-C5B } & -1.3(2) \\ \text { N2B-C2B-N4B-C6B } & 0.6(4) \\ \text { C3B-C2B-N4B-C6B } & -176.8(2) \\ \text { C1B-N1B-C4B-O1B } & 176.86(19) \\ \text { C1B-N1B-C4B-C3B } & -1.8(3) \\ \text { N3B-C3B-C4B-O1B } & -1.6(4) \\ \text { C2B-C3B-C4B-O1B } & -176.5(2) \\ \text { N3B-C3B-C4B-N1B } & 177.0(2) \\ \text { C2B-C3B-C4B-N1B } & 2.0(3) \\ \text { N2B-C1B-N5B-C11B } & 0.3(3) \\ \text { N1B-C1B-N5B-C11B } & -179.74(18) \\ \text { C3B-N3B-C5B-N4B } & -1.0(3) \\ \text { C2B-N4B-C5B-N3B } & 1.5(3) \\ \text { C6B-N4B-C5B-N3B } & 177.4(2) \\ \text { C9B-O2B-C6B-N4B } & -153.74(17) \\ \text { C9B-O2B-C6B-C7B } & -28.9(2) \\ \text { C5B-N4B-C6B-O2B } & -113.2(2) \\ \text { C2B-N4B-C6B-O2B } & 61.7(3) \\ \text { C5B-N4B-C6B-C7B } & 128.3(2) \\ \text { C2B-N4B-C6B-C7B } & -56.9(3) \\ \text { O2B-C6B-C7B-C8B } & 36.8(2) \\ \text { N4B-C6B-C7B-C8B } & 156.41(19) \\ & \end{array}$




$\begin{array}{ll}\mathrm{C} 6 \mathrm{~A}-\mathrm{C} 7 \mathrm{~A}-\mathrm{C} 8 \mathrm{~A}-\mathrm{O} 3 \mathrm{~A} & 79.6(2) \\ \mathrm{C} 6 \mathrm{~A}-\mathrm{C} 7 \mathrm{~A}-\mathrm{C} 8 \mathrm{~A}-\mathrm{C} 9 \mathrm{~A} & -36.8(2) \\ \mathrm{C} 6 \mathrm{~A}-\mathrm{O} 2 \mathrm{~A}-\mathrm{C} 9 \mathrm{~A}-\mathrm{C} 8 \mathrm{~A} & -10.4(2) \\ \mathrm{C} 6 \mathrm{~A}-\mathrm{O} 2 \mathrm{~A}-\mathrm{C} 9 \mathrm{~A}-\mathrm{C} 10 \mathrm{~A} & 113.18(18) \\ \text { O3A-C8A-C9A-O2A } & -88.60(19) \\ \mathrm{C} 7 \mathrm{~A}-\mathrm{C} 8 \mathrm{~A}-\mathrm{C} 9 \mathrm{~A}-\mathrm{O} 2 \mathrm{~A} & 29.7(2) \\ \mathrm{O} 3 \mathrm{~A}-\mathrm{C} 8 \mathrm{~A}-\mathrm{C} 9 \mathrm{~A}-\mathrm{C} 10 \mathrm{~A} & 151.55(18) \\ \mathrm{C} 7 \mathrm{~A}-\mathrm{C} 8 \mathrm{~A}-\mathrm{C} 9 \mathrm{~A}-\mathrm{C} 10 \mathrm{~A} & -90.2(2) \\ \mathrm{O} 2 \mathrm{~A}-\mathrm{C} 9 \mathrm{~A}-\mathrm{C} 10 \mathrm{~A}-\mathrm{O} 4 \mathrm{~A} & 165.83(17) \\ \mathrm{C} 8 \mathrm{~A}-\mathrm{C} 9 \mathrm{~A}-\mathrm{C} 10 \mathrm{~A}-\mathrm{O} 4 \mathrm{~A} & -76.0(2) \\ \mathrm{C} 1 \mathrm{~A}-\mathrm{N} 5 \mathrm{~A}-\mathrm{C} 11 \mathrm{~A}-\mathrm{O} 5 \mathrm{~A} & -87.4(2) \\ \mathrm{C} 1 \mathrm{~A}-\mathrm{N} 5 \mathrm{~A}-\mathrm{C} 11 \mathrm{~A}-\mathrm{C} 12 \mathrm{~A} & 156.64(19) \\ \mathrm{C} 14 \mathrm{~A}-\mathrm{O} 5 \mathrm{~A}-\mathrm{C} 11 \mathrm{~A}-\mathrm{N} 5 \mathrm{~A} & -148.25(16) \\ \mathrm{C} 14 \mathrm{~A}-\mathrm{O} 5 \mathrm{~A}-\mathrm{C} 11 \mathrm{~A}-\mathrm{C} 12 \mathrm{~A} & -26.8(2) \\ \text { N5A-C11A-C12A-C13A } & 154.32(17) \\ \text { O5A-C11A-C12A-C13A } & 35.6(2) \\ \text { C17A-O6A-C13A-C12A } & 167.04(19) \\ \mathrm{C} 17 \mathrm{~A}-\mathrm{O} 6 \mathrm{~A}-\mathrm{C} 13 \mathrm{~A}-\mathrm{C} 14 \mathrm{~A} & -78.6(2) \\ \text { C11A-C12A-C13A-O6A } & 89.7(2) \\ \text { C11A-C12A-C13A-C14A } & -30.6(2) \\ \text { C11A-O5A-C14A-C15A } & 128.97(18) \\ \text { C11A-O5A-C14A-C13A } & 7.2(2) \\ \text { O6A-C13A-C14A-O5A } & -102.90(18) \\ \text { C12A-C13A-C14A-O5A } & 15.1(2) \\ \text { O6A-C13A-C14A-C15A } & 136.89(18) \\ \text { C12A-C13A-C14A-C15A } & -105.08(19) \\ \text { C16A-O7A-C15A-C14A } & -173.67(18) \\ \text { O5A-C14A-C15A-O7A } & -66.7(2) \\ \text { C13A-C14A-C15A-O7A } & 51.9(2) \\ \end{array}$

\begin{tabular}{|c|c|}
\hline $\mathrm{C} 6 \mathrm{~B}-\mathrm{C} 7 \mathrm{~B}-\mathrm{C} 8 \mathrm{~B}-\mathrm{O} 3 \mathrm{~B}$ & $85.4(2)$ \\
\hline $\mathrm{C} 6 \mathrm{~B}-\mathrm{C} 7 \mathrm{~B}-\mathrm{C} 8 \mathrm{~B}-\mathrm{C} 9 \mathrm{~B}$ & $-30.0(2)$ \\
\hline $\mathrm{C} 6 \mathrm{~B}-\mathrm{O} 2 \mathrm{~B}-\mathrm{C} 9 \mathrm{~B}-\mathrm{C} 10 \mathrm{~B}$ & $131.8(2)$ \\
\hline $\mathrm{C} 6 \mathrm{~B}-\mathrm{O} 2 \mathrm{~B}-\mathrm{C} 9 \mathrm{~B}-\mathrm{C} 8 \mathrm{~B}$ & $8.8(2)$ \\
\hline $\mathrm{O} 3 \mathrm{~B}-\mathrm{C} 8 \mathrm{~B}-\mathrm{C} 9 \mathrm{~B}-\mathrm{O} 2 \mathrm{~B}$ & $-103.77(1$ \\
\hline $\mathrm{C} 7 \mathrm{~B}-\mathrm{C} 8 \mathrm{~B}-\mathrm{C} 9 \mathrm{~B}-\mathrm{O} 2 \mathrm{~B}$ & $14.3(2)$ \\
\hline $\mathrm{O} 3 \mathrm{~B}-\mathrm{C} 8 \mathrm{~B}-\mathrm{C} 9 \mathrm{~B}-\mathrm{C} 10 \mathrm{~B}$ & $137.8(2)$ \\
\hline $\mathrm{C} 7 \mathrm{~B}-\mathrm{C} 8 \mathrm{~B}-\mathrm{C} 9 \mathrm{~B}-\mathrm{C} 10 \mathrm{~B}$ & $-104.1(2)$ \\
\hline $\mathrm{O} 2 \mathrm{~B}-\mathrm{C} 9 \mathrm{~B}-\mathrm{C} 10 \mathrm{~B}-\mathrm{O} 4 \mathrm{~B}$ & $-68.9(3)$ \\
\hline $\mathrm{C} 8 \mathrm{~B}-\mathrm{C} 9 \mathrm{~B}-\mathrm{C} 10 \mathrm{~B}-\mathrm{O} 4 \mathrm{~B}$ & $49.4(3)$ \\
\hline $\mathrm{C} 1 \mathrm{~B}-\mathrm{N} 5 \mathrm{~B}-\mathrm{C} 11 \mathrm{~B}-\mathrm{O} 5 \mathrm{~B}$ & $-93.7(2)$ \\
\hline $\mathrm{C} 1 \mathrm{~B}-\mathrm{N} 5 \mathrm{~B}-\mathrm{C} 11 \mathrm{~B}-\mathrm{C} 12 \mathrm{~B}$ & $151.2(2)$ \\
\hline $\mathrm{C} 14 \mathrm{~B}-\mathrm{O} 5 \mathrm{~B}-\mathrm{C} 11 \mathrm{~B}-\mathrm{N} 5 \mathrm{~B}$ & $-163.87(16$ \\
\hline $\mathrm{C} 14 \mathrm{~B}-\mathrm{O} 5 \mathrm{~B}-\mathrm{C} 11 \mathrm{~B}-\mathrm{C} 12 \mathrm{~B}$ & $-41.2(2)$ \\
\hline $\mathrm{N} 5 \mathrm{~B}-\mathrm{C} 11 \mathrm{~B}-\mathrm{C} 12 \mathrm{~B}-\mathrm{C} 13 \mathrm{~B}$ & $162.67(18)$ \\
\hline $\mathrm{O} 5 \mathrm{~B}-\mathrm{C} 11 \mathrm{~B}-\mathrm{C} 12 \mathrm{~B}-\mathrm{C} 13 \mathrm{~B}$ & $43.9(2)$ \\
\hline $\mathrm{C} 17 \mathrm{~B}-\mathrm{O} 6 \mathrm{~B}-\mathrm{C} 13 \mathrm{~B}-\mathrm{C} 12 \mathrm{~B}$ & $172.60(18)$ \\
\hline $\mathrm{C} 17 \mathrm{~B}-\mathrm{O} 6 \mathrm{~B}-\mathrm{C} 13 \mathrm{~B}-\mathrm{C} 14 \mathrm{~B}$ & $-74.3(2)$ \\
\hline $\mathrm{C} 11 \mathrm{~B}-\mathrm{C} 12 \mathrm{~B}-\mathrm{C} 13 \mathrm{~B}-\mathrm{O} 6 \mathrm{~B}$ & $88.8(2)$ \\
\hline $\mathrm{C} 11 \mathrm{~B}-\mathrm{C} 12 \mathrm{~B}-\mathrm{C} 13 \mathrm{~B}-\mathrm{C} 14 \mathrm{~B}$ & $-30.0(2)$ \\
\hline $\mathrm{C} 11 \mathrm{~B}-\mathrm{O} 5 \mathrm{~B}-\mathrm{C} 14 \mathrm{~B}-\mathrm{C} 15 \mathrm{~B}$ & $146.08(17)$ \\
\hline $\mathrm{C} 11 \mathrm{~B}-\mathrm{O} 5 \mathrm{~B}-\mathrm{C} 14 \mathrm{~B}-\mathrm{C} 13 \mathrm{~B}$ & $21.5(2)$ \\
\hline $\mathrm{O} 6 \mathrm{~B}-\mathrm{C} 13 \mathrm{~B}-\mathrm{C} 14 \mathrm{~B}-\mathrm{O} 5 \mathrm{~B}$ & $-109.88(1$ \\
\hline $\mathrm{C} 12 \mathrm{~B}-\mathrm{C} 13 \mathrm{~B}-\mathrm{C} 14 \mathrm{~B}-\mathrm{O} 5 \mathrm{~B}$ & $6.4(2)$ \\
\hline $\mathrm{O} 6 \mathrm{~B}-\mathrm{C} 13 \mathrm{~B}-\mathrm{C} 14 \mathrm{~B}-\mathrm{C} 15 \mathrm{~B}$ & $128.32(19)$ \\
\hline $\mathrm{C} 12 \mathrm{~B}-\mathrm{C} 13 \mathrm{~B}-\mathrm{C} 14 \mathrm{~B}-\mathrm{C} 15 \mathrm{~B}$ & $-115.5(2)$ \\
\hline $\mathrm{C} 16 \mathrm{~B}-\mathrm{O} 7 \mathrm{~B}-\mathrm{C} 15 \mathrm{~B}-\mathrm{C} 14 \mathrm{~B}$ & $-175.77(1$ \\
\hline $\mathrm{O} 5 \mathrm{~B}-\mathrm{C} 14 \mathrm{~B}-\mathrm{C} 15 \mathrm{~B}-\mathrm{O} 7 \mathrm{~B}$ & $-69.6(2)$ \\
\hline $\mathrm{C} 13 \mathrm{~B}-\mathrm{C} 14 \mathrm{~B}-\mathrm{C} 15 \mathrm{~B}-\mathrm{O} 7 \mathrm{~B}$ & $50.2(2)$ \\
\hline
\end{tabular}

Hydrogen-bond geometry $\left(\AA,{ }^{\circ}\right)$

\begin{tabular}{lllll}
\hline$D-\mathrm{H} \cdots A$ & $D-\mathrm{H}$ & $\mathrm{H} \cdots A$ & $D \cdots A$ & $D-\mathrm{H} \cdots A$ \\
\hline $\mathrm{N} 1 A-\mathrm{H} 1 A \cdots \mathrm{N} 3 B$ & 0.88 & 1.92 & $2.789(2)$ & 170 \\
$\mathrm{O} 3 A-\mathrm{H} 3 A \cdots \mathrm{O} 5 A^{\mathrm{i}}$ & 0.84 & 2.07 & $2.897(2)$ & 167 \\
$\mathrm{O} 4 A-\mathrm{H} 4 A \cdots \mathrm{O} 3 B^{\mathrm{ii}}$ & 0.84 & 2.01 & $2.847(2)$ & 178 \\
$\mathrm{~N} 5 A-\mathrm{H} 5 A \cdots \mathrm{O} 1 B$ & 0.88 & 2.23 & $3.058(2)$ & 157 \\
$\mathrm{C} 5 A-\mathrm{H} 5 A 1 \cdots \mathrm{O} 1 B^{\mathrm{iii}}$ & 0.95 & 2.63 & $3.284(3)$ & 126 \\
$\mathrm{C} 7 A-\mathrm{H} 7 A 1 \cdots \mathrm{N} 2 A$ & 0.99 & 2.46 & $3.172(3)$ & 128 \\
$\mathrm{C} 8 A-\mathrm{H} 8 A \cdots \mathrm{O} 7 A^{\mathrm{i}}$ & 1.00 & 2.39 & $3.316(3)$ & 153 \\
$\mathrm{C} 12 A-\mathrm{H} 12 A \cdots \mathrm{O} 1 A^{\mathrm{iv}}$ & 0.99 & 2.61 & $3.432(3)$ & 141 \\
$\mathrm{C} 12 A-\mathrm{H} 12 B \cdots \mathrm{O} 1 B$ & 0.99 & 2.55 & $3.426(3)$ & 147 \\
$\mathrm{C} 16 A-\mathrm{H} 16 A \cdots \mathrm{O} 4 A^{\mathrm{v}}$ & 0.98 & 2.47 & $3.401(3)$ & 158 \\
$\mathrm{C} 16 A-\mathrm{H} 16 B \cdots \mathrm{O} 6 A^{\mathrm{v}}$ & 0.98 & 2.54 & $3.222(3)$ & 127 \\
$\mathrm{C} 16 A-\mathrm{H} 16 C \cdots \mathrm{O} 2 A^{\mathrm{vi}}$ & 0.98 & 2.50 & $3.356(3)$ & 146 \\
$\mathrm{C} 17 A-\mathrm{H} 17 A \cdots \mathrm{O} 3 A^{\mathrm{vi}}$ & 0.98 & 2.65 & $3.610(3)$ & 168 \\
$\mathrm{C} 17 A-\mathrm{H} 17 B \cdots \mathrm{O} 2 A^{\mathrm{iv}}$ & 0.98 & 2.60 & $3.573(3)$ & 175 \\
$\mathrm{~N} 1 B-\mathrm{H} 1 B \cdots \mathrm{N} 3 A^{\mathrm{vi}}$ & 0.88 & 1.94 & $2.808(2)$ & 166
\end{tabular}




\begin{tabular}{|c|c|c|c|c|}
\hline $\mathrm{O} 3 B-\mathrm{H} 3 B^{\cdots} \cdots \mathrm{O} 5 B^{\mathrm{vii}}$ & 0.84 & 1.99 & $2.817(2)$ & 169 \\
\hline $\mathrm{O} 4 B-\mathrm{H} 4 B \cdots \mathrm{N} 2 B$ & 0.84 & 2.38 & $3.180(3)$ & 158 \\
\hline $\mathrm{N} 5 B-\mathrm{H} 5 B \cdots \mathrm{O} 1 A^{\mathrm{vi}}$ & 0.88 & 2.19 & $3.027(2)$ & 159 \\
\hline $\mathrm{C} 5 B-\mathrm{H} 5 B 1 \cdots \mathrm{O} 1 A$ & 0.95 & 2.60 & $3.269(3)$ & 127 \\
\hline $\mathrm{C} 8 B-\mathrm{H} 8 B \cdots \mathrm{O} 7 B^{\text {vii }}$ & 1.00 & 2.49 & $3.363(3)$ & 146 \\
\hline $\mathrm{C} 11 B-\mathrm{H} 11 B \cdots \mathrm{O} 4 B$ & 1.00 & 2.59 & $3.251(3)$ & 124 \\
\hline $\mathrm{C} 12 B-\mathrm{H} 12 C \cdots \mathrm{O} 1 A^{\mathrm{vi}}$ & 0.99 & 2.55 & $3.363(3)$ & 140 \\
\hline $\mathrm{C} 12 B-\mathrm{H} 12 D^{\cdots} \mathrm{O} 1 B^{v}$ & 0.99 & 2.45 & $3.424(3)$ & 167 \\
\hline $\mathrm{C} 14 B-\mathrm{H} 14 B \cdots \mathrm{O} 4 B$ & 1.00 & 2.61 & $3.272(3)$ & 123 \\
\hline $\mathrm{C} 17 B-\mathrm{H} 17 E^{\cdots} \mathrm{O} 2 B^{\mathrm{v}}$ & 0.98 & 2.48 & $3.456(3)$ & 176 \\
\hline
\end{tabular}

Symmetry codes: (i) $x-1, y, z$; (ii) $-x+1, y+1 / 2,-z$; (iii) $x-1, y, z-1$; (iv) $x, y, z+1$; (v) $x+1, y, z$; (vi) $x+1, y, z+1$; (vii) $x, y, z-1$. 